\title{
Multiscale modelling of tumour growth and therapy: the influence of vessel normalisation on chemotherapy
}

\author{
TOMÁS ALARCÓN†*, MARKUS R. OWEN $\ddagger$, HELEN M. BYRNE $\ddagger$ and PHILIP K. MAINIף
}

\author{
$\dagger$ Bioinformatics Unit, Department of Computer Science, University College London, \\ Gower Street, London WC1E 6BT, UK \\ $\ddagger$ School of Mathematical Sciences, Centre for Mathematical Medicine, University of Nottingham, \\ Nottigham NG7 2RD, UK \\ १ Mathematical Institute, Centre for Mathematical Biology, University of Oxford, \\ 24-29 St Giles', Oxford OX1 3LB, UK
}

(Received 11 June 2006; in final form 14 July 2006)

\begin{abstract}
Following the poor clinical results of antiangiogenic drugs, particularly when applied in isolation, tumour biologists and clinicians are now turning to combinations of therapies in order to obtain better results. One of these involves vessel normalisation strategies. In this paper, we investigate the effects on tumour growth of combinations of antiangiogenic and standard cytotoxic drugs, taking into account vessel normalisation. An existing multiscale framework is extended to include new elements such as tumour-induced vessel dematuration. Detailed simulations of our multiscale framework allow us to suggest one possible mechanism for the observed vessel normalisation-induced improvement in the efficacy of cytotoxic drugs: vessel dematuration produces extensive regions occupied by quiescent (oxygen-starved) cells which the cytotoxic drug fails to kill. Vessel normalisation reduces the size of these regions, thereby allowing the chemotherapeutic agent to act on a greater number of cells.
\end{abstract}

Keywords: Hybrid cellular automaton; VEGF; Vessel dematuration; Antiangiogenic therapy

\section{Introduction}

Cancer cells depend on sufficient nutrient supply for survival and, therefore, growth of a (solid) tumour requires access to blood vessels. Cancer cells can also acquire an invasive phenotype enabling them to enter the circulation and eventually establish metastases. Thus, the onset of angiogenesis (the process by which new vessels are generated from existing ones) appears to mark the transition of a solid tumour from a mostly harmless, localised lesion into a potentially fatal, systemic disease. In this context, antiangiogenic drugs were anticipated to provide a breakthrough in anticancer therapy. In spite of the high expectations raised by early successes in animal experiments, these drugs have fared poorly in clinical trials. When administered alone, antiangiogenic drugs achieve modest objective responses but their impact on life expectancy and long-term survival has been disappointingly poor (see [14] and references therein).

*Corresponding author. Email: t.alarcon@cs.ucl.ac.uk

Computational and Mathematical Methods in Medicine ISSN 1748-670X print/ISSN 1748-6718 online () 2006 Taylor \& Francis

http://www.tandf.co.uk/journals

DOI: $10.1080 / 10273660600968994$ 
In spite of this failure of antiangiogenic drugs as a stand-alone therapy, the results of combinations of antiangiogenic drugs with conventional chemotherapy offer a more optimistic perspective. For example, a drug called bevacizumab, an antibody against a potent angiogenic substance secreted (among others) by cancer cells called vascular endothelial growth factor (VEGF), in combination with cytotoxic drugs yielded a significant increase in survival in colorectal cancer patients [18]. These results support the predictions of Teicher [29], who anticipates that the combination of antigiogenic drugs with conventional chemotherapy would yield optimal effects, as this combination targets two tumour compartments (cancer cells and endothelial cells). According to Teicher [29], antiangiogenic drugs should always increase the effects of cytotoxic drugs.

These observations can be considered somehow paradoxical, as, in principle, antiangiogenic therapy removes (or prevents formation of) tumour blood vessels, thereby disabling the access route the cytotoxic drugs use to reach the tumour [14]. Thus, it could be expected that less drug would reach the tumour reducing its overall cell killing effect. Essentially, the same could be expected for radiotherapy. Antiangiogenic therapy would remove blood vessels which, in turn, would produce extensive areas of hypoxia (low oxygen levels) within the tumour. Furthermore, hypoxia is known to antagonise radiation therapy. In fact, there exist studies showing that antiangiogenic therapy may compromise the outcomes of both chemo- and radio-therapy [14].

The mechanisms by which antiangiogenic therapy improve cytotoxic drug efficiency are not completely understood. However, work by Jain et al. [14,30,32] provides strong evidence to support the involvement of antiangiogenic therapy-induced vessel normalization which may be explained as follows. Tumour vasculature is known to exhibit a number of abnormalities in comparison to its normal counterpart. Normal vasculature has a welldefined anatomical structure essential for its functionality. Tumour vessels are usually immature and lack this structure. Consequently, they are leaky and not quiescent, unlike normal vessels. Additionally, immature tumour vessels are prone to collapse under the pressure of the growing tumour population. These structural abnormalities yield, in turn, abherrant blood flow and oxygen delivery with spatial and temporal heterogeneities. These features combine to produce an abnormal micro-environment in which increased hypoxia (low levels of oxygen), which renders cells resistant to chemo- and radio-therapy, and abherrant tumour blood flow, pose severe barriers to drug delivery and efficacy [12].

In this context, it seems that fixing (i.e. normalising) the tumour vasculature could help to re-establish normal delivery of drugs and oxygen thus allowing more cancer cells to receive effective concentrations of both. This is the rationale behind normalisation strategies. Angiogenesis, in both pathological and physiological situations, is controlled by an increase in angiogenic promoters (e.g. VEGF) over their inhibitor counterparts. In normal conditions, this imbalance will eventually be counterbalanced. In a pathological setting, the imbalance persists. Therefore, restoring the balance using antiangiogenic substances may result in a more normal-looking vasculature. However, excessive doses of antiangiogenic drugs may hinder the effect of conventional therapy. It has been observed in several studies that antiangiogenic therapies antagonise chemo- or radio-therapy by reducing oxygenation and drug delivery. This indicates that there is a delicate balance between normalisation and (excessive) vessel regression, implying that extreme care is needed in the selection of dose and schedule [14].

Recent modelling work addressing the effect of vessel dematuration on tumour growth was recently presented in Ref. [4]. A cellular automaton model was developed to analyse the effect of the growing tumour on the vasculature which was incorporated with simple models 
of angiogenesis, vessel co-option and vessel collapse (as a consequence of vessel dematuration). Bartha and Rieger [4] concluded that vascular density might not be an appropriate indicator of tumour prognosis, as is generally believed. Instead, their model suggests that tumour progression, at least in its early stages, is strongly determined by the vascular density of the original host tissue.

In Ref. [25] Ribba et al. incorporated a model of vessel dematuration upon co-option by the growing tumour into a model designed to assess typical clinical protocols of doxorubicin treatment of Non-Hodgkin lymphomas. The dematuration model used in Ref. [25] was extremely simple: whenever a vessel was co-opted by the tumour its radius would be assigned a value drawn from a randomly generated Gaussian distribution. The rationale for this model was that when a vessel is co-opted a process is triggered whereby the normal mechanisms dictating vessel behaviour cease to act. In this context, assigning a random value to the radii of co-opted vessels might be a reasonable first approximation. However, this model did not incorporate any of the mechanisms of vessel dematuration upon engulfment. In addition, a thorough investigation of the effects of vessel dematuration was not carried out.

Although Teicher [29] appears to have reached the right conclusion, her "linear" reasoning, which is typical of attempts by biologists to establish a non-quantitative theoretical framework [9], neglects many of the complex interactions between the different elements involved in the process of tumour growth. Our aim in this paper is to place Teicher's theoretical ideas in a quantitative framework which allows us first to check their validity and then to explore possible mechanisms involved in the improvement of the efficacy of cytotoxic drugs administered together with antiangiogenic drugs. This will be done by adapting the multiscale model of vascular tumour growth developed by us $[1,3,5]$ to allow for vessel dematuration upon co-option by a growing tumour.

This paper is organised as follows. Section 2 provides an overview of the multiscale framework used in this paper. Section 3 summarises results presented previously in Ref. [16] which help us to understand the new results reported in this paper. In section 4 we compare the responses of tumours with normal and cancerous vasculatures to treatment with cytotoxic drug. In section 5 we summarise the biological background to tumour-induced vessel dematuration and explain how we incorporate this phenomenon within our multiscale framework. In section 6, we present simulation results that illustrate how a tumour's evolution depends on whether the underlying vasculature is normal or pathological and whether vessel dematuration occurs. In section 7 , we investigate the effect of treating a tumour with a combination of chemotherapy and an anti-VEGF receptor antibody which antagonises VEGF and the effect that it has on the outcome of chemotherapy. Finally, in section 8 we discuss our results, model limitations and future research directions.

\section{Summary of the multiscale model}

In this section, we describe briefly the elements and organisation of the multiscale model. This framework has been described in detail in Ref. [3,5]. We refer the reader to these previous papers for a thorough account of the model formulation.

Our model is based on the hybrid cellular automaton concept which has been used to model several aspects of tumour development (see $[1,6,21])$. We extend this approach to account not only for the presence of a diffusive substance (such as oxygen or glucose) as in previous papers, but also to include intracellular and tissue-scale phenomena, and the coupling between them. To this end, we have organised our model into three layers: vascular, 


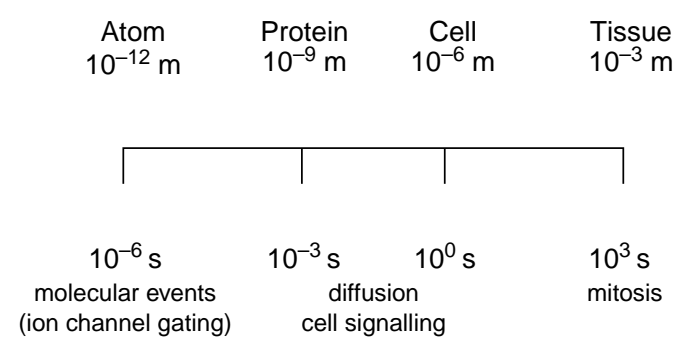

Figure 1. Time and length scales involved in our model [11].

cellular and intracellular, which correspond, respectively, to the tissue, cellular and intracellular TLSs (figures 1 and 2).

In the tissue layer, we deal with the structural properties of the vascular network and blood flow [1]. We consider a hexagonal vascular network (similar to the one observed in liver). Each vessel is assumed to undergo structural adaptation (i.e. changes in radius) in response to different stimuli until the network reaches a quasi-equilibrium state. We then compute the blood flow rate, the pressure drop and the haematocrit (i.e. relative volume of red blood cells) distribution in each vessel. The dynamics of the vascular layer and the cellular layer are coupled by the transport of blood-borne oxygen into the tissue. This process is modelled by a reaction-diffusion equation. The distribution of haematocrit in the vessels acts as the source of oxygen, whereas the cell distribution defines the (spatially distributed) sink of oxygen.

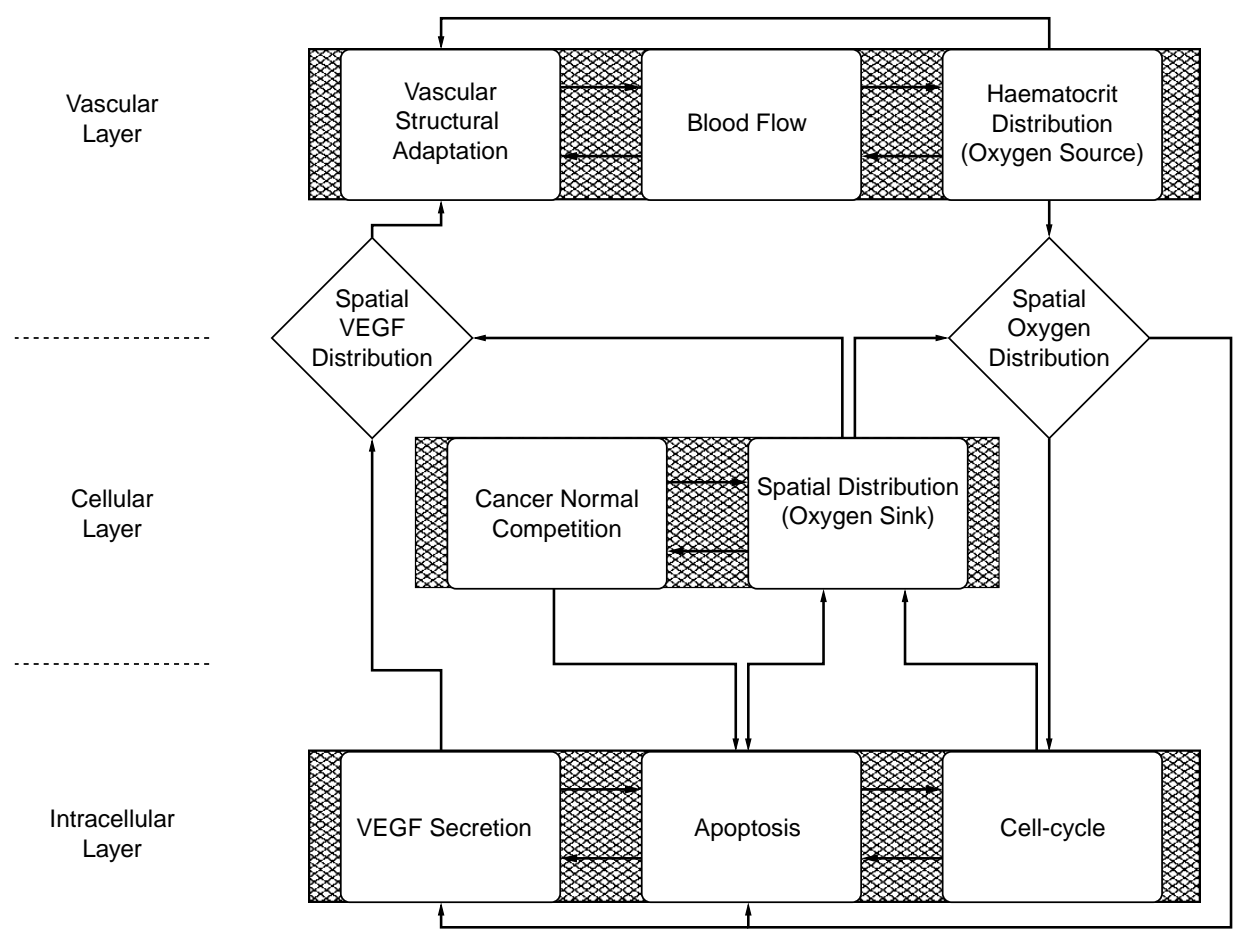

Figure 2. Diagrammatic representation of the layer structure of our model. 
In the cellular layer, we focus on normal and cancer cells which are treated as individual elements. These two populations compete for space and resources. Competition between the two cell types is introduced by a simple rule, which couples the cellular to the intracellular layer. Apoptosis (programmed cell death) is controlled by the expression of p53 (whose dynamics are dealt with in the intracellular layer): when the level of p53 in a cell exceeds some threshold the cell undergoes apoptosis. However, this threshold depends on the local spatial distribution of cells, which links the spatial distribution (cellular layer) with the apoptotic process (intracellular layer).

Processes included in the intracellular layer are cell division, apoptosis and VEGF secretion. In this layer, we use ordinary differential equations (ODEs) to model the relevant biochemistry. One issue we focus on is how the external conditions modulate the dynamics of these intracellular phenomena and, in particular, how the level of extracellular oxygen affects the division rate, the expression of p53 (which regulates apoptosis) and the production of VEGF. Since the spatial distribution of oxygen depends on both the spatial distribution of cells (cellular layer) and on the distribution of haematocrit (vascular layer), these processes at the intracellular level are linked to the behaviour of the other two layers: cell proliferation and apoptosis alter the spatial distribution of the cells (figure 2); the cellular and intracellular layers modulate the process of vascular structural adaptation through another transport process: diffusion of VEGF into the tissue and its absorption by the endothelial cells (ECs) lining the vessels.

Since the main focus of this paper is on vessel normalisation, brief mathematical descriptions of the cellular and intracellular layers are presented in the Appendix while a detailed description of the vascular layer is given in section 3 .

\section{Structural adaptation in normal and cancerous vasculature}

Pries et al. have constructed a model $[23,24]$ in which normal vessels adapt their radii in response to a number of different stimuli. In a vessel of radius $R(t)$ we have:

$$
\frac{\mathrm{d} R}{\mathrm{~d} t}=S_{\mathrm{tot}} R
$$

where $S_{\text {tot }}$ is the total stimulus, corresponding to the sum of the different stimuli which we describe briefly now.

First there is the haemodynamic stimulus, $S_{\mathrm{h}}$, through which the vessels adapt to blood flow conditions. The main signals involved here appear to be wall shear stress (WSS), $\tau_{w}$, and pressure, $\mathbf{P}$ [26], with increased WSS typically increasing the vessel radius and increased pressure decreasing it [24]. Accordingly, Pries et al. [24] postulate the following form for the haemodynamic stimulus, $S_{\mathrm{h}}$ :

$$
S_{\mathrm{h}}=\log \left(\tau_{w}+\tau_{r}\right)-k_{p} \log \left(\tau_{e}(\mathbf{P})\right)
$$

where $\tau_{r}$ is a constant introduced to avoid singular behaviour for low WSS, $k_{p}$ is a constant and $\tau_{e}(\mathbf{P})$ is a set-point level of WSS corresponding to the actual value of the intravascular pressure [24].

The second stimulus is the so-called metabolic stimulus $\left(S_{\mathrm{m}}\right)$ which describes how vessels adapt to the metabolic needs of the surrounding tissue. Pries et al. [23] considered the 
following functional form for $S_{\mathrm{m}}$ :

$$
S_{\mathrm{m}}=k_{m} \log \left(1+\frac{\dot{Q}_{r}}{\dot{Q} H}\right)
$$

where $k_{m}$ is a constant, $\dot{Q}_{r}$ is a reference blood flow and $H$ is the haematocrit. In Ref. [3], a modification of equation (3) was proposed to account for the effect that VEGF, $V$, has on the vasculature (VEGF is produced by nutrient-deprived cells). In Ref. [3], the constant $k_{m}$ was replaced by a function of $V$ :

$$
k_{m}(V)=k_{m}^{0}\left(1+\frac{V}{V_{0}+V}\right)
$$

where $k_{m}^{0}$ and $V_{0}$ are constants.

The third stimulus is actually a pair of stimuli, the so-called conducted stimuli, and consists of signals generated by the vessels that propagate either downstream or upstream. They are assumed to be necessary to maintain a fully functional vascular system. These signals are usually emitted under stress conditions, and therefore are closely related to the metabolic stimulus described above. Although many underlying biological details of these signalling mechanisms are unknown, the downstream stimulus is believed to be transmitted by a chemical which is released into the blood (a good candidate seems to be ATP released by red blood cells under hypoxic conditions) and thereby carried downstream by the flow. The upstream transmission of information seems to be along the vessel walls, perhaps by the spread of changes in membrane potential through gap junctions [24].

The intensity of the downstream stimulus, $S_{\mathrm{d}}$, is assumed to depend on the current of the signalling chemical along a particular vessel (vessel "1", say). If the vessels upstream (vessels "2" and "3") of vessel 1 are irrigating hypoxic regions, they will receive signals from the tissue in the form of secreted VEGF. If the concentration of VEGF in vessel 2 or 3 is positive, then it will produce a (constant) amount of signalling chemical, $\rho_{0}$. The chemical will enter vessel 1 and its current along vessel 1 will be (due to mass conservation):

$$
J_{1}=\rho_{1}(V) \dot{Q}_{1}+\rho_{2}(V) \dot{Q}_{2}
$$

where $\rho_{i}(V)=\rho_{0}$ if $V \neq 0$ in vessel $i=1,2$ and $\rho_{i}(V)=0$ if $V=0$ in vessel $i=1,2$. Following [24], we write:

$$
S_{\mathrm{d}}=\log \left(1+\frac{J_{1}}{\dot{Q}+\dot{Q}_{\mathrm{ref}}}\right)
$$

where $\dot{Q}_{\text {ref }}$ is a constant introduced to avoid singular behaviour.

The upstream stimulus, $S_{\mathrm{u}}$, is assumed to depend on a signal produced by vessels in hypoxic regions $(V>0)$. The "amount" of signal produced is assumed to be proportional to the length of the vessel, $L_{\mathrm{s}}$. As in Ref. [24], we further assume that the upstream signal dissipates as it propagates, modelling this process with exponential decay. At a given node of the network, the current of upstream stimulus produced by each "outgoing" vessel 
(defined as one such that the corresponding current has a negative value) is given by:

$$
J_{\mathrm{c}}^{0}=L_{\mathrm{s}} \mathrm{e}^{-L_{\mathrm{s}} / L}
$$

where $L$ is a constant.

The total current, $J_{\mathrm{c}}$, is the sum over all the outgoing vessels at a given node of the corresponding values of $J_{\mathrm{c}}^{0}$. The upstream stimulus at each of the incoming vessels at the corresponding node is given by Ref. [24]:

$$
S_{\mathrm{u}}=k_{m} k_{c} \frac{J_{\mathrm{c}}}{J_{\mathrm{c}}+J_{0}}
$$

where $J_{0}$ is a constant and $k_{m}=k_{m}(V)$ as in equation (4).

Combining the above assumptions we deduce that the total stimulus, $S_{\text {tot }}$, is given by

$$
S_{\mathrm{tot}}=S_{\mathrm{h}}+S_{\mathrm{m}}+S_{\mathrm{d}}+S_{\mathrm{u}}-k_{s}
$$

where $S_{\mathrm{h}}, S_{\mathrm{m}}, S_{\mathrm{d}}$ and $S_{\mathrm{u}}$ are defined by equations (2)-(8) and $k_{s}$ is a constant corresponding to the so-called shrinking stimulus, which, according to [23], corresponds to the tendency of vessels to shrink in the absence of growth factors or other external stimuli.

The above stimuli together account for many functional and structural properties of normal vascular networks [24]. Tumour vessels, in contrast, are far from normal. The welldefined anatomical structure and orderly spatial distribution of normal vessels, that produce an efficient and functional vascular system, are replaced by leaky vessels that lack most of the anatomical features of their normal counterparts and exhibit a more random-looking spatial distribution of vessels. This yields more unstructured and inefficient vascular networks. In this context, it would be useful to know which, if any, of the adaptation mechanisms mentioned above for normal vessels are likely to be active and which are absent in cancer vessels.

This issue was studied by Maini et al. in Ref. [16]. The strategy there was to "deconstruct" the normal vasculature, deactivating in turn each of the stimuli affecting adaptation in normal vessels. Using our multiscale model, tumour growth was simulated for a range of structural adaptation mechanisms and the results compared with qualitative structural features known to be exhibited by solid tumours. Based on this study, we concluded that the conducted stimuli are most likely to be absent in tumour vasculature [16]. In view of these results, a vasculature for which $S_{\mathrm{tot}}=S_{\mathrm{h}}+S_{\mathrm{m}}+S_{\mathrm{d}}+S_{\mathrm{u}}-k_{s}$ will hereafter be referred to as normal whereas a vasculature for which $S_{\text {tot }}=S_{\mathrm{h}}+S_{\mathrm{m}}-k_{s}$ will be referred to as cancerous.

\subsection{Simulation results: normal and cancerous vasculatures}

Before considering the effect of therapy, it is useful to present simulations showing how the different structural adaptation mechanisms affect the growth properties of our model tumours [16]. Figures 3-5 show results for tumours perfused by normal and cancerous vasculatures. There are two interesting features to note. One concerns the size of the tumour supported by the vascular networks: being more efficient, the normal vasculature gives rise to a larger tumour than its cancerous counterpart. Taken in isolation this result would suggest that normalising the tumour vasculature might not be an efficient therapeutic strategy.

However, figure 5(b) shows a second interesting result, namely the time evolution of the number of quiescent cancer cells for the normal and cancerous vasculatures. We observe that, 

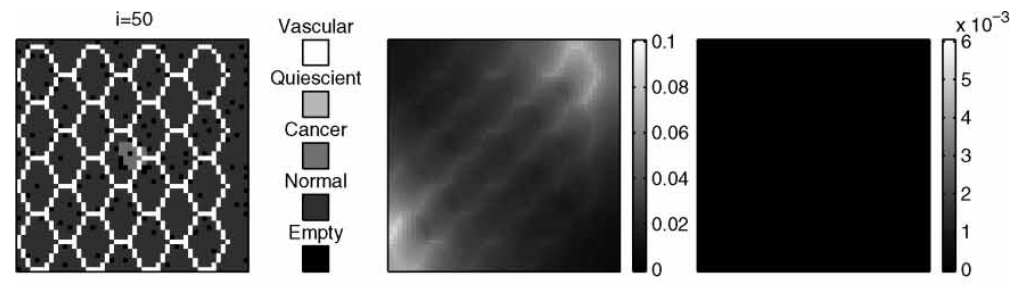

$\mathrm{i}=100$
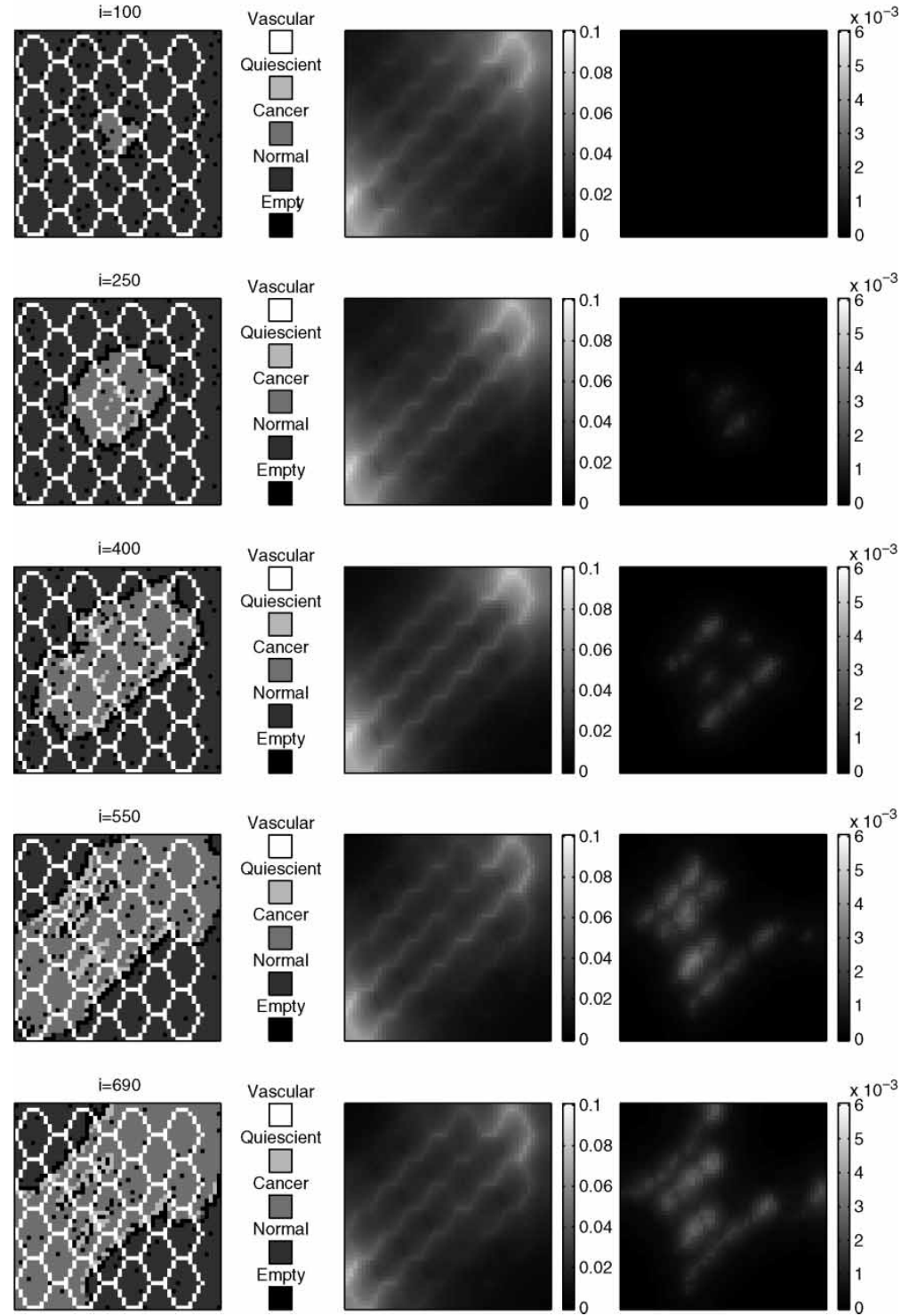

Figure 3. Snapshots of the dynamics of our multiscale model when the structural adaptation mechanism has $S_{\mathrm{tot}}=S_{\mathrm{h}}+S_{\mathrm{m}}-k_{s}$ with $k_{m}=k_{m}(V)$ defined by equation (4). This simulation corresponds to tumour growth with a cancerous vasculature. No drugs are included in this simulation. Left panels show the spatial distribution of cells, middle panels the distribution of oxygen and right panels the distribution of VEGF. 

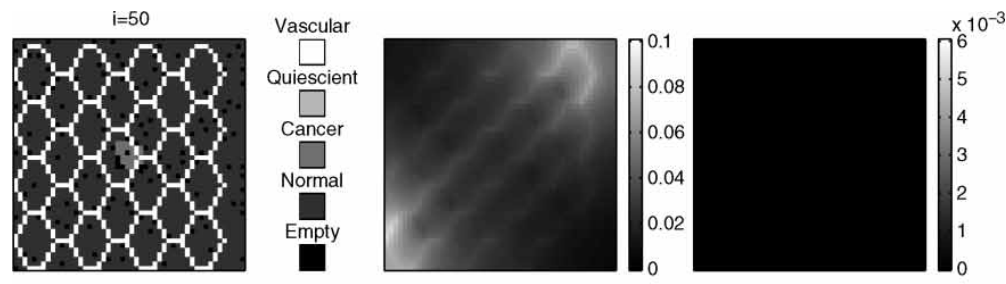

$\mathrm{i}=100$
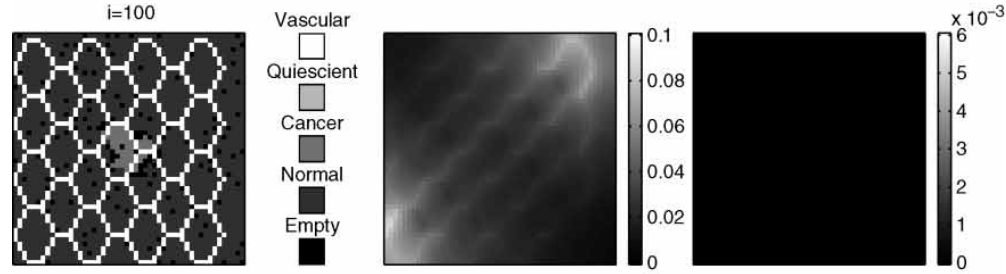

$\mathrm{i}=250$
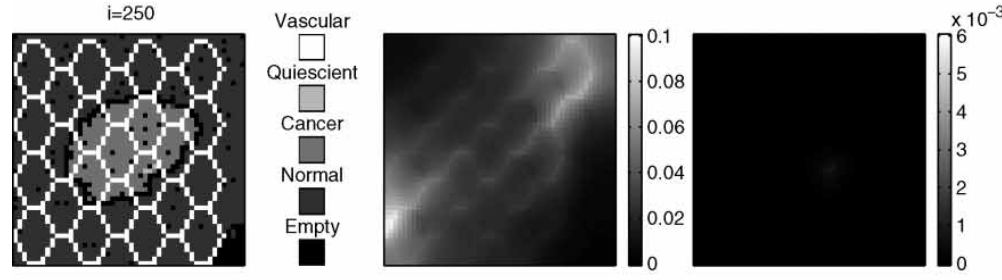

$i=400$
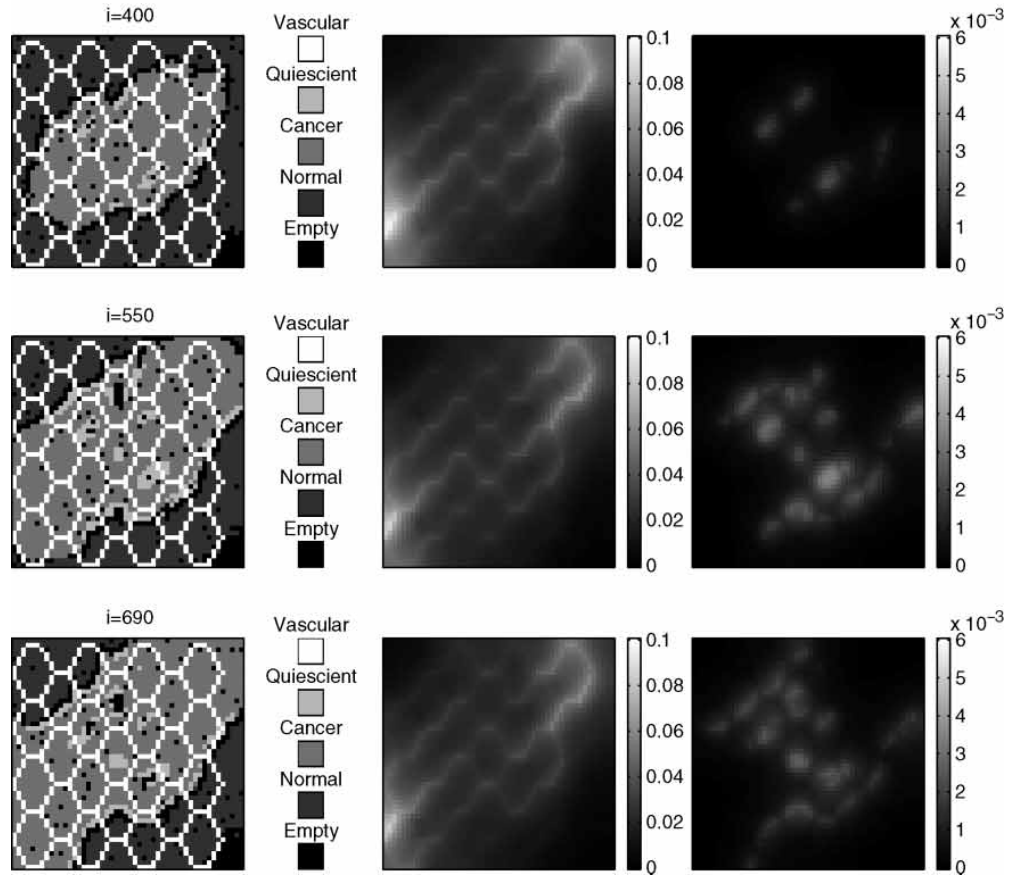

Figure 4. Snapshots of the dynamics of our multiscale model when the structural adaptation mechanism has $S_{\mathrm{tot}}=S_{\mathrm{h}}+S_{\mathrm{m}}+S_{\mathrm{d}}+S_{\mathrm{u}}-k_{s}$ with $k_{m}=k_{m}(V)$ defined by equation (4). This simulation corresponds to tumour growth with a normal vasculature. No drugs are included in this simulation. Left panels show the spatial distribution of cells, middle panels the distribution of oxygen and right panels the distribution of VEGF. 

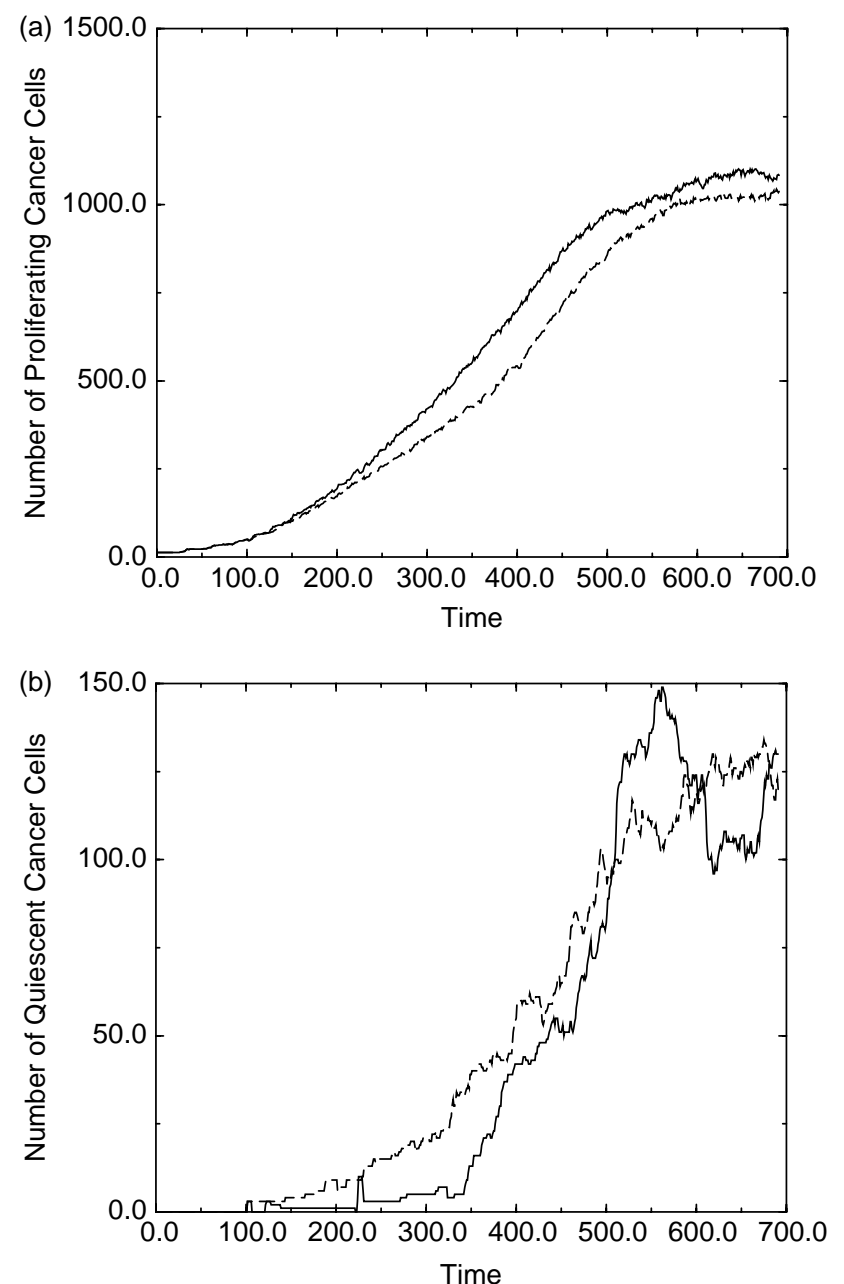

Figure 5. Time evolution of the number of (a) proliferating cancer cells and (b) quiescent cancer cells for the simulations presented in figures 3 and 4. Solid and dashed lines correspond to normal and cancerous vasculature, respectively.

most of the time, the quiescent population supported by the cancerous vasculature is larger than that supported by the normal vasculature. This fact, which is also due to the higher efficiency of the normal vasculature, might have important implications for the outcome of combined therapy for the following reasons. In our model, cell quiescence is induced by hypoxia $\dagger$. Since most cytotoxic drugs target proliferating cells and hypoxia is know to delay (and even halt) progression through the cell cycle, hypoxia reduces drug efficacy and, thereby, contributes to drug resistance. For similar reasons, hypoxic regions are also resistant to radiotherapy [14].

This result suggests how normalising tumour vessels might improve a tumour's response to chemo- or radio-therapy: by reducing the size of the resistant (quiescent) compartment.

$\dagger$ This means that, as far as our model is concerned, regions occupied by quiescent cells can be identified with hypoxic regions. 
Although normalisation could increase the number of proliferating cancer cells, they are susceptible to the cytotoxic drugs and therefore should be destroyed by the chemotherapy.

\section{Simulation results: inclusion of a cytotoxic drug}

As a first attempt to assess whether vessel normalisation might improve the performance of chemotherapeutic agents, we ran simulations of our multiscale model with a blood-borne cytotoxic drug as detailed in Section A.4. The cytotoxic drug is administered in the form of two bolus injections at times $t=150$ and $t=200$. The corresponding results are shown in figures 6 and 7.

Figures 6(a) and 7(a) show, respectively, the time evolution of the number of proliferating cancer cells with and without chemotherapy and for normal and cancerous vasculatures. We see that chemotherapy reduces the number of proliferating cells for both types of vasculature, although, in agreement with our previous predictions [16], chemotherapy seems to be only slightly more effective for the normal vasculature than for the cancerous one. Further, this difference appears to be too small to justify the apparent success of combined cytotoxic/antiangiogenic strategies.

\section{Vessel dematuration}

The simulations presented in figure 6 do not account for the process of tumour-induced vessel dematuration and its dependence on tumour-produced VEGF. The inclusion of some of these features into our model is the subject of the present section.

It is now widely recognised that tumours interact with the host vasculature. One way in which they do this is via angiogenesis, a process which has been widely studied by the theoretical community (see the excellent review by Mantzaris et al. [17] and references $[19,28]$ for more recent models of angiogenesis including blood flow). With the exception of [4], one aspect of tumour-vasculature coupling that has received much less attention is tumour-induced vessel dematuration. As tumours grow, "healthy" vessels of the host organism are engulfed by the tumour mass and a process of dematuration, which includes loss of wall vessel structure and increased leakiness, is triggered $[13,33]$. Experimental data indicate that this process is due to overexpression of a protein called Angiopoietin 2 (hereafter referred to as Ang2) in the ECs of engulfed vessels. However, the effect of Ang2 on the fate of the engulfed vessels is contextual: if VEGF levels are high, Ang2 contributes to increased sprouting, i.e. it promotes the onset of angiogenesis. If, by contrast, VEGF levels are low, Ang2 destabilises the vessels [33].

In our simulations, therefore, we now distinguish three types of vessels according to the mechanism that controls their structural adaptation. Normal vessels are those surrounded by a majority of normal cells. They adapt according to the normal total stimulus (see section 3). By contrast, a vessel surrounded by a majority of cancer cells is considered as co-opted or engulfed and its behaviour depends on the local concentration of VEGF $\dagger$.

Most of the biomolecular details of vessel dematuration are unknown and quantitative information is scarce. Thus, in order to proceed further, we formulate a rather simple model which retains what it is currently believed to occur. We introduce a vessel maturity variable,

$\dagger$ For a discussion of what is meant by the local VEGF concentration of a vessel, see [3]. 

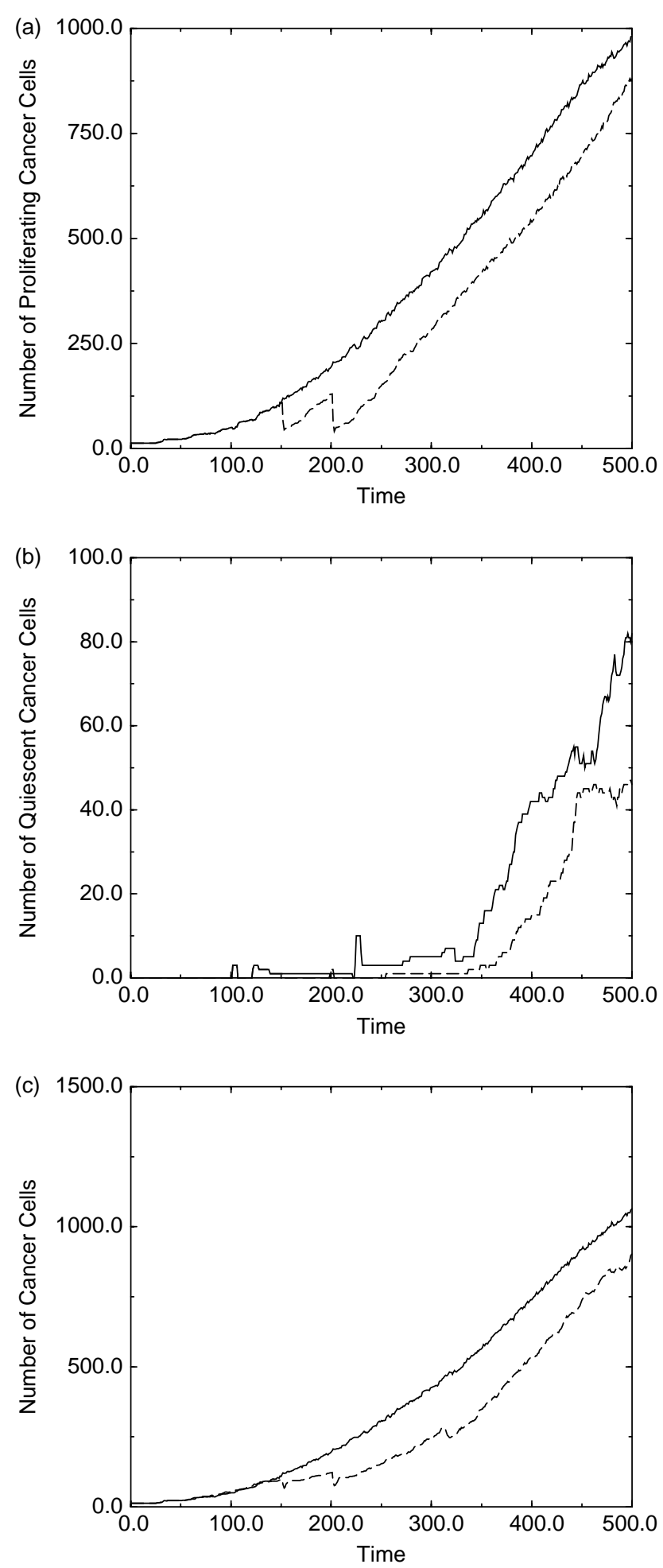
$M$, which characterises the final fate of the vessel in the presence of VEGF: $M=0$ corresponds to dematuration, $M \neq 0$ corresponds to a normal vessel or to an angiogenic tumour vessel.

Similar to equation (1), which describes the dynamics of vessel radius in response to haemodynamical and external stimuli, we formulate an equation for the variable $M$ :

$$
\frac{\mathrm{d} M}{\mathrm{~d} t}=\alpha\left(V-V_{\mathrm{c}}\right) M-\beta M^{3}
$$

where $\alpha$ and $\beta$ are positive constants. The steady state values of $M$ are $M_{1}=0$ and $M_{2}=\left(\alpha\left(V-V_{\mathrm{c}}\right) / \beta\right)^{1 / 2}$. We note that $M_{1}$ is unstable and $M_{2}$ is stable for $V>V_{\mathrm{c}}$. Hence, for $V>V_{\mathrm{c}}$ mature/angiogenic vessels will be stable whereas immature/collapsing vessels will be stable for $V<V_{\mathrm{c}}$, where $V_{\mathrm{c}}$ is the critical concentration of VEGF at which an engulfed vessel switches from one status to the other.

We assume further that the characteristic timescale for the dynamics of $M$ is much shorter than the structural adaptation timescale, and hence that $M$ is in (quasi-)steady state. This assumption allows us to reduce the number of unknown parameters in the model, as, under this assumption, vessel fate is essentially controlled by a single parameter, $V_{\mathrm{c}}$ (equation (10)).

We will assume that mature and immature vessels, i.e. vessels with $M=M_{1}$ or $M=M_{2}$, respectively, undergo structural adaptation according to different stimuli:

$$
\frac{\mathrm{d} R}{\mathrm{~d} t}=\delta_{M, M_{1}}\left(S_{\mathrm{c}}-\eta\right) R+\delta_{M, M_{2}} S_{\mathrm{n}} R,
$$

where $\delta_{x, y}$ represents the Kronecker delta and $S_{\mathrm{n}}$ and $S_{\mathrm{c}}$ are the normal and cancerous adaptation stimuli, respectively:

$$
\begin{gathered}
S_{\mathrm{c}}=S_{\mathrm{h}}-k_{s} \\
S_{\mathrm{n}}=S_{\mathrm{h}}+S_{\mathrm{m}}-k_{s}
\end{gathered}
$$

The parameter $\eta$ corresponds to the collapse experienced by cancerous vessels under the pressure of the growing tumour. Such collapse is a consequence of the loss of structural stability of the cancerous vessels.

Equation (11) incorporates the two vascular fates described above. The first term on the right hand side corresponds to the low-VEGF behaviour of the engulfed vessels and is intended to describe vessel collapse where the change in vessel radius is the product of two opposing forces: haemodynamic forces exerted by the blood on the vessel wall $\left(S_{\mathrm{c}}\right)$ and pressure exerted by the tumour cells $(\eta)$.

The second term on the right hand side of equation (11) describes an abnormal vessel (see section 3) in which the metabolic stimulus depends on the local VEGF concentration, thus accounting in an effective manner for angiogenic effects.

Figure 6. Time evolution of the number of (a) proliferating cancer cells, (b) quiescent cancer cells and (c) total number of cancer cells under normal vasculature. Solid lines correspond to growth of an untreated tumour, dashed lines to tumour growth treated with a cytotoxic drug. $h_{\theta}=0.07$. Chemotherapy is administered in the form of two bolus injections at $t=150$ and $t=200$. The amount of vessel drug is taken to be proportional to $1-H$ (i.e. the fluid fraction of blood contained in a given vessel). Anti-VEGF drug is not included in this simulation. 

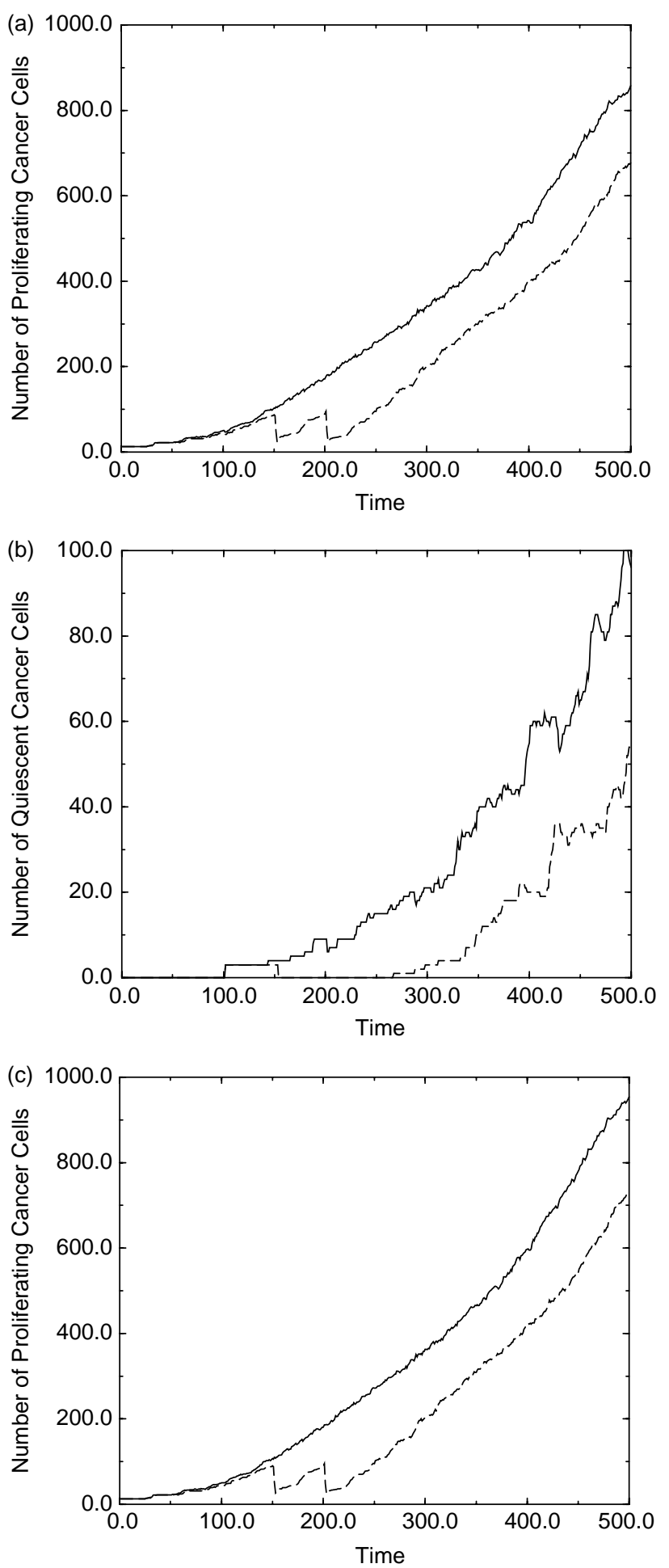
The algorithm to determine vessel behaviour is as follows:

i) All vessels are initially labelled NORMAL.

ii) The numbers of cancer and normal cells within one lattice site distance from the vessel are counted. If the former exceed the latter the vessel is termed CO-OPTED, otherwise it remains NORMAL. Vessel co-option is reversible, i.e. if this condition ceases to hold, a co-opted vessel is re-labelled NORMAL.

iii) Normal vessels undergo structural adaptation according to equation (1) with $S_{\text {tot }}=$ $S_{\mathrm{h}}+S_{\mathrm{m}}+S_{\mathrm{d}}+S_{\mathrm{u}}-k_{s}$ (see section 3$)$.

iv) Co-opted vessels undergo structural adaptation according to equations (11) and (12) with $M(V)$ determined by the associated stable steady state solution of equation (10).

\section{Simulation results: normal vasculature vs. dematuration}

Here, we discuss the differences between the behaviour observed in our model under a normal(ised) vasculature and a vasculature generated according to equation (11) and the effect that vessel dematuration has on the tumour's response to cytotoxic drugs.

Figures 8 and 9 show simulations performed without chemotherapy with a vasculature generated according to equation (11), i.e. taking into account the effects of vessel dematuration as described in section 5 . We observe that vessel co-option and dematuration lead to the formation of extensive regions populated by quiescent cells (figure 8): these are much larger than those observed in simulations with a normal vasculature (figure 9(b)). Since quiescent cells are, in general, resistant to cytotoxic drugs (which target mostly proliferating cells), this could be an important factor for chemotherapy efficacy.

Figure 9 shows oscillations in both the proliferating and quiescent subpopulations. Mechanistically, these oscillations are produced by the interplay between vessel engulfment, regression and angiogenic response. When vessels are engulfed they initially undergo regression under the pressure of the tumour cells. This reduces the oxygen supply, leading to hypoxia and cell quiescence. These cells then produce VEGF which causes a switch in vessel behaviour from shrinking to angiogenic. In our model, this induces a local increase in vessel diameter, which enables the cells to recommence their progress through the cell cycle. This interplay between the tumour mass and the vasculature is depicted in figure 10 where the dynamics of the vascular network under this rather complex adaptation mechanism is shown.

From these results we conclude that (i) vessel dematuration could be an important factor in the complex dynamical behaviour of tumours, and (ii) the appearance of extensive regions of hypoxia might hinder the action of cytotoxic drugs, which act preferentially on proliferating cells.

To clarify further the role of hypoxic regions populated by quiescent cells, we ran simulations with a cytotoxic drug administered via two bolus injections at times $t=150$ and $t=200$ (figures 11 and 12). Figure 12(a),(b) show the time evolution of the total number of

Figure 7. Time evolution of the number of (a) proliferating cancer cells and (b) quiescent cancer cells and (c) total number of cancer cells under cancerous vasculature. Solid lines correspond to growth of an untreated tumour, dashed lines to tumour growth treated with a cytotoxic drug. $h_{\theta}=0.07$. Chemotherapy is administered in the form of two bolus injections at $t=150$ and $t=200$. The amount of vessel drug is taken to be proportional to $1-H$ (i.e. the fluid fraction of blood contained in a given vessel). Anti-VEGF drug is not included in this simulation. 

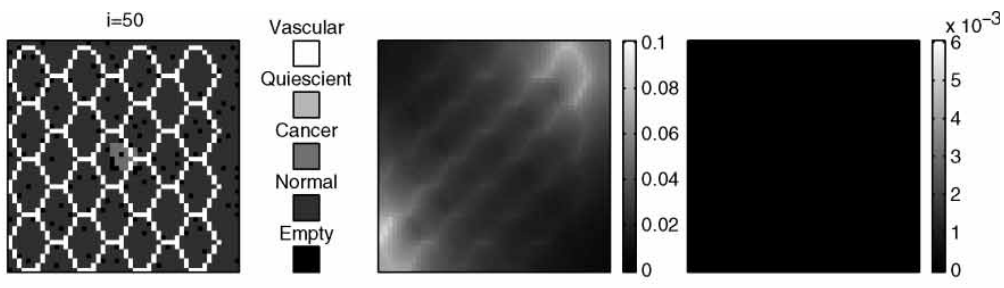

$\mathrm{i}=150$
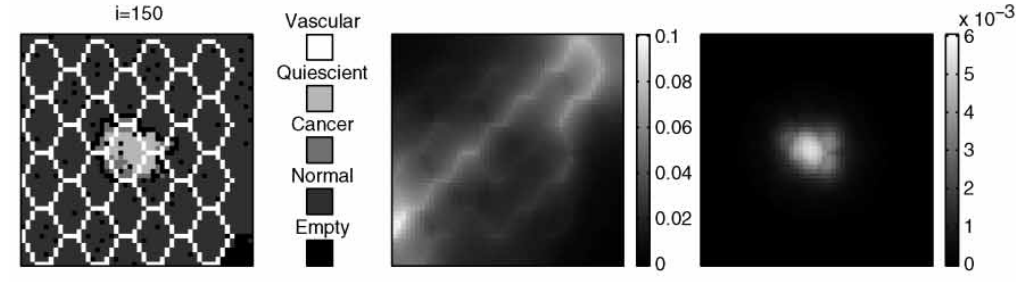

$\mathrm{i}=250$
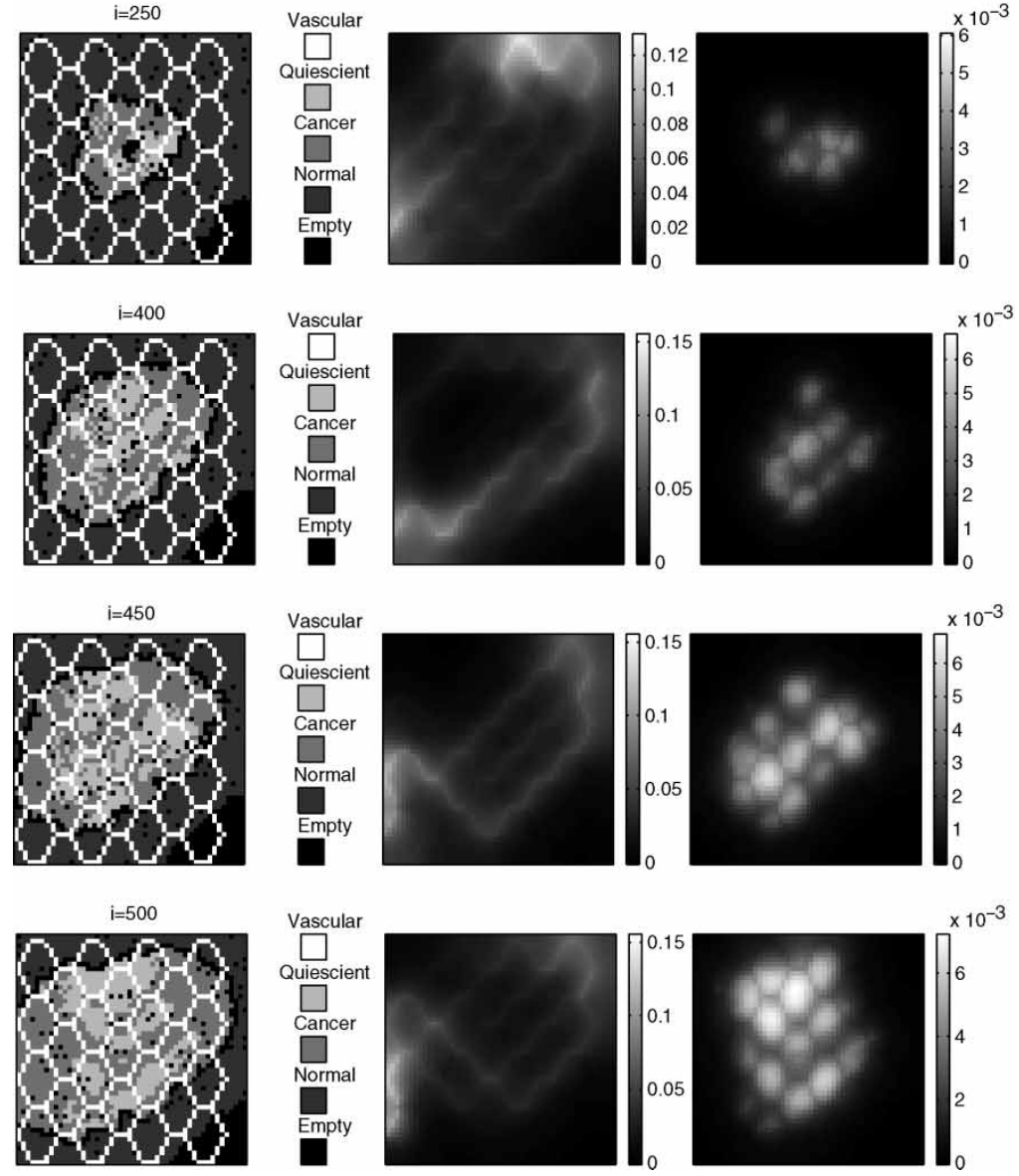

Figure 8. Snapshots of the dynamics of our multiscale model under the structural adaptation mechanism given by equation (11) with $\eta=5 k_{m}^{0}$ and $k_{m}$ given by equation (4). Cytotoxic drug and anti-VEGF drug are not included in this simulation. Left panels show the spatial distribution of cells, middle panels the distribution of oxygen and right panels the distribution of VEGF. 

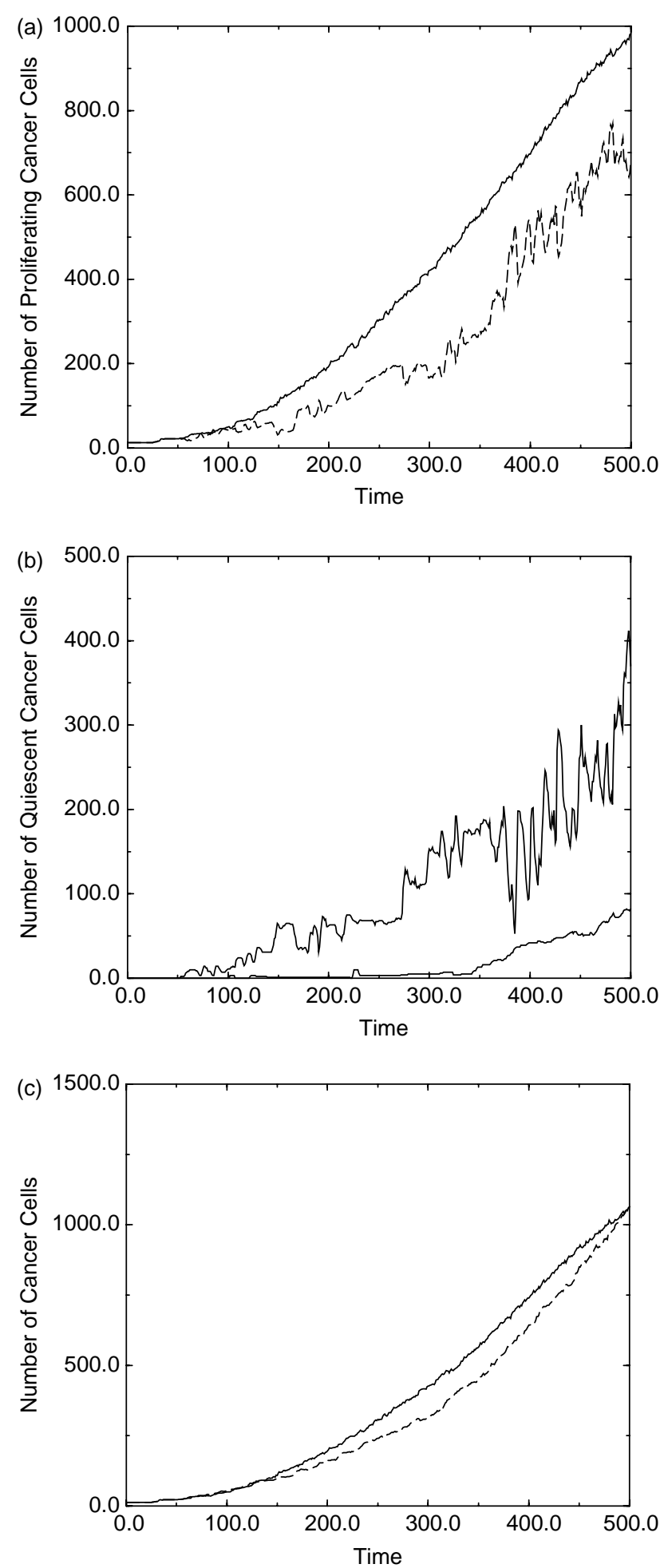

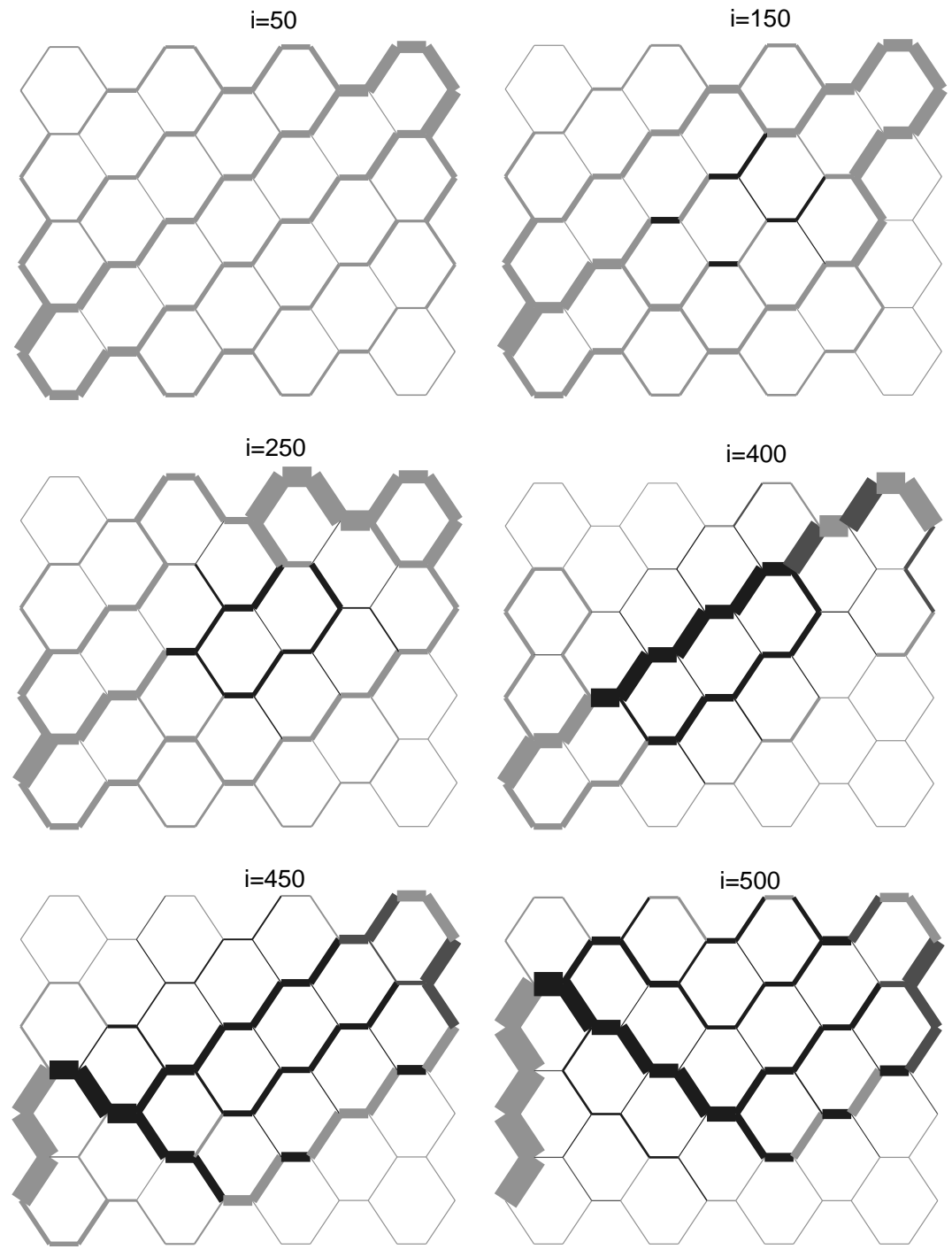

Figure 10. Snapshots of the dynamics of the vasculature when the structural adaptation mechanism is given by equation (11) with $k_{m}$ given by equation (4). These results correspond to the simulations shown in figure 8 . Brown corresponds to normal vessels, red to co-opted-regressing vessels and blue to co-opted-angiogenic vessels. Line width is proportional to the haematocrit in each vessel. $\eta=5 k_{m}^{0}$. Cytotoxic drug and anti-VEGF drug are not included in this simulation.

cancer cells. We note that the efficacy of the drug deteriorates markedly when the vessels undergo co-option and dematuration: in terms of both the total number of cells killed by the drug and the time the cancer needs to recover after the injection, the cytotoxic drug is less effective when the vessels are subject to co-option and dematuration.

Figure 9. Time evolution of the number of (a) proliferating cancer cells and (b) quiescent cancer cells for the simulations shown in figure 8 . Solid lines correspond to normal vasculature, dashed lines to a vasculature generated according to equation (11). eta $=5 k_{m}^{0}$. Cytotoxic drug and anti-VEGF drug are not included in this simulation. 

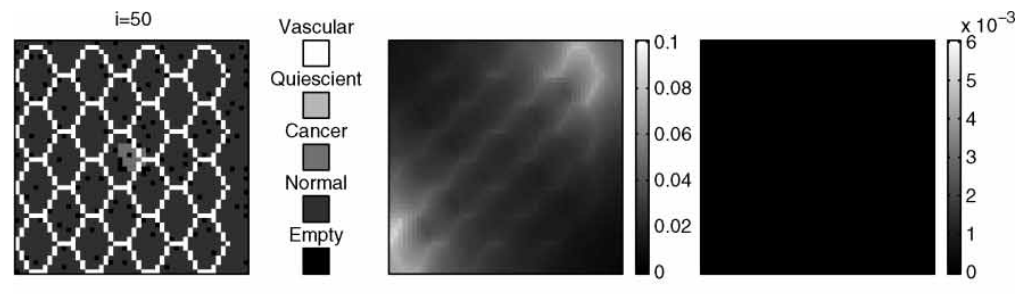

$\mathrm{i}=150$
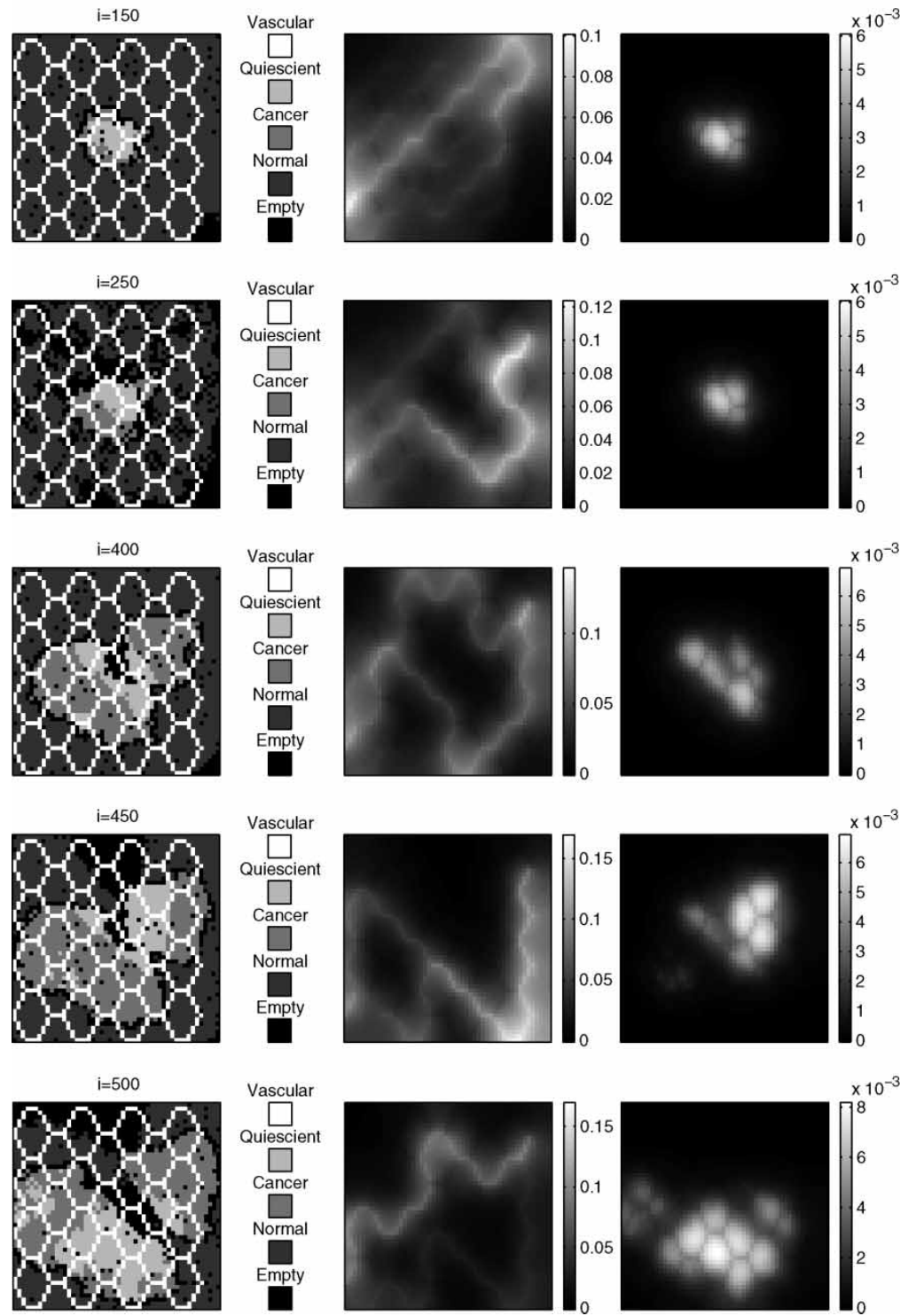

Figure 11. Snapshots of the dynamics of our multiscale model when the structural adaptation mechanism is given by equation (11) and $k_{m}$ by equation (4) and a cytotoxic drug has been administered. $h_{\theta}=0.07$. Amount of vessel drug is taken to be proportional to $1-H$ (i.e. the fluid fraction of blood contained in a given vessel). $\eta=5 k_{m}^{0}$. AntiVEGF drug is not included in this simulation. Left panels show the spatial distribution of cells, middle panels the distribution of oxygen and right panels the distribution of VEGF. 

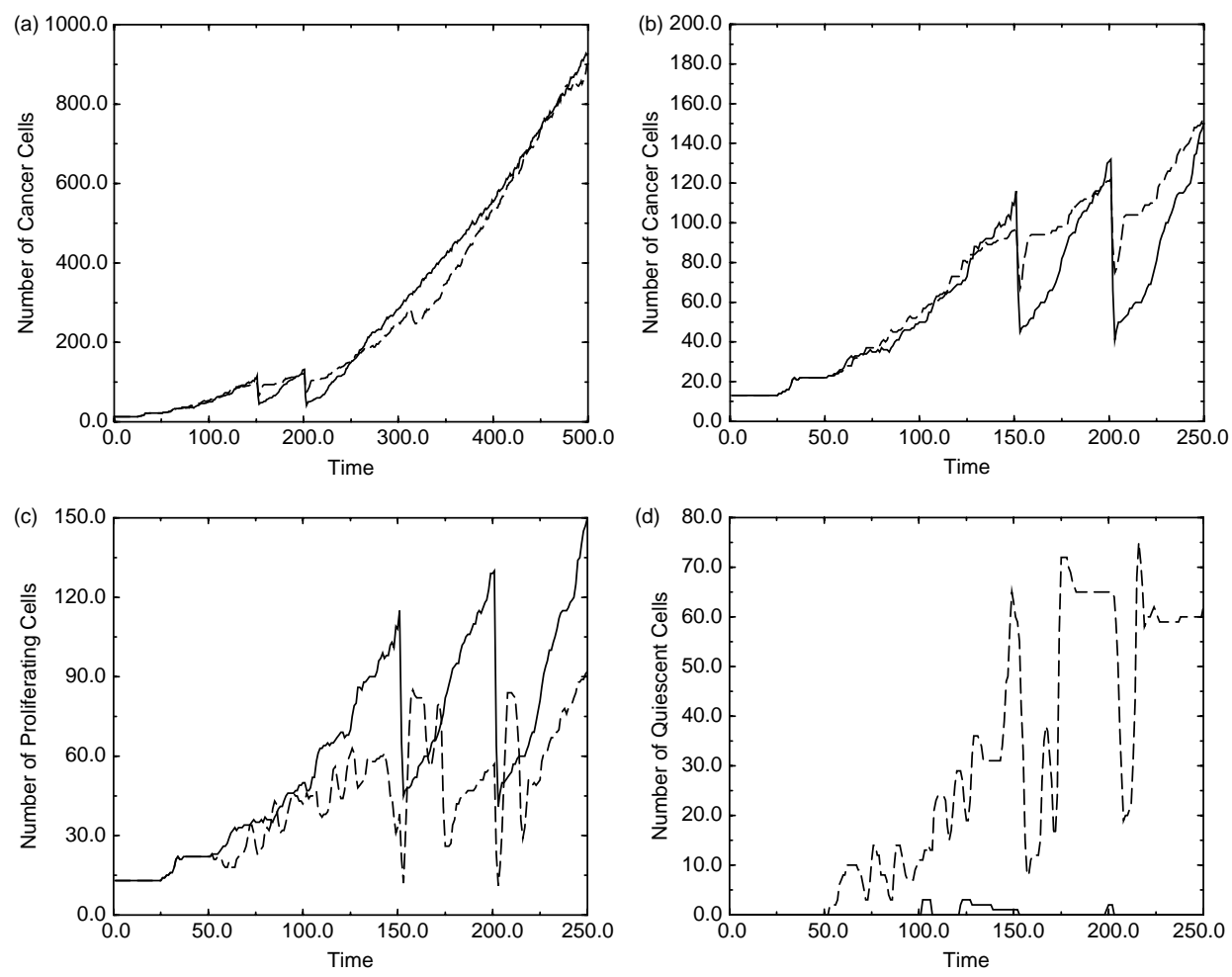

Figure 12. Time evolution of the total number of cancer cells ((a) and (b) close-up), number of proliferating cells (c) and number of quiescent cancer cells (d). Solid and dashed lines correspond to simulations for a normal vasculature and a vasculature generated according to equation (11), respectively. Cytotoxic drug is administered in the form of two bolus injections at time $t=150$ and $t=200 . h_{\theta}=0.07 . \eta=5 k_{m}^{0}$. Anti-VEGF drug is not included in this simulation.

A more detailed picture of how this occurs can be obtained from the time courses of the proliferating and quiescent subpopulations (figure 12(c),(d), respectively). Figure 12(d) shows that the numbers of proliferating and quiescent cells drop dramatically after drug administration. However, whereas proliferating cells are killed by the therapy, the reduction in quiescent cell numbers, which are immune to the action of the cytotoxic drug, is due to quiescent cells starting to proliferate again (note the sudden increase in proliferating cell numbers just after the injections).

It is important to point out that the results presented in the section 5 for the "cancerous vasculature" differ from those involving vessel dematuration. This difference is due to the fact that vessel dematuration acts locally within the network whereas in the cancerous vasculature the upstream and downstream stimuli are inactivated over the entire network.

\section{Vessel normalisation}

Following the observation that a combination of antiangiogenic therapy with either chemoor radio-therapy is effective, Jain et al. have investigated what mechanisms might be responsible for such synergistic behaviour. In a number of papers [14,30,32], they proposed 
that antiangiogenic therapy, either in the form of an antibody against VEGF or a VEGF receptor blockade, leads to vascular normalisation, i.e. the tumour vasculature, normally disorganised and inefficient, partially recovers and acquires the structure of normal vascular beds. Winkler et al. [32] have conducted experiments in brain tumours in which VEGF receptor 2 (VEGFR2) blockade, by means of a specific antibody against VEGFR2, creates a so-called normalisation window, i.e. a period of time during which the vasculature acquires a more normal structure. This partial normalisation improves tumour oxygenation and increases the efficacy of radiation therapy.

Tong et al. [30] have found evidence that vascular normalisation following VEGFR2 blockade improves the delivery of chemotherapy to the tumour mass by decreasing the interstitial fluid pressure.

\subsection{Anti-VEGFR therapy}

In order to see whether our model can reproduce the results of Winkler et al. [32] and what insight our model can provide regarding the mechanisms involved, we introduce into our multiscale model a new element, a diffusible anti-VEGFR drug, i.e. a specific antibody against VEGFR that inhibits the effect of VEGF by competitively binding its receptor. To keep the model as simple as possible, we assume that only ECs have VEGFR. This assumption is accurate in normal tissue, but there is some evidence that cancer cells might also respond to VEGF [14].

The mathematical description of the anti-VEGFR drug is similar to that used for VEGF (Section A.1) and the cytotoxic drug (Section A.4), i.e. a diffusion equation is used to describe its extracellular concentration $R$. We will assume that the anti-VEGFR is delivered to the tumour via the vasculature. The equation for $R$ takes the form:

$$
0=D_{R} \nabla^{2} R-h_{R}\left(R_{\mathrm{vess}}-R\right)-h_{R}^{\prime} R-\lambda_{R} R
$$

where $D_{R}$ denotes the assumed constant diffusion coefficient of the anti-VEGFR antibody, $h_{R}$ the rate at which it is transported across the vessel wall and $h_{R}^{\prime}$ is the rate at which VEGF binds to ECs on the surface of the vessel walls. The calculation of the anti-VEGFR concentration within the vascular network, $R_{\text {vess }}$, is strongly related to the computation of the haematocrit, $H$ (see section 2.1). In particular, we assume that the drug is soluble in plasma and, therefore, advected with the liquid fraction of blood, i.e. $1-H$. Accordingly, we assume that the concentration of drug within each vessel is given by $R_{\text {adm }}(1-H)$ where $R_{\text {adm }}$ is the administered dose. This is analogous to how we model the chemotherapeutic drug.

The presence of the anti-VEGFR antibody alters the form of the metabolic stimulus for vascular adaptation, in particular the form of equation (4), which assumes a MichaelisMenten kinetics for VEGF binding to the VEGFR on the surface of the ECs. Anti-VEGFR is a competitive inhibitor of VEGF as it competes for binding to the VEGFR. Consequently, the expression for $k_{m}(V)$ in equation (4) should be changed to [15]:

$$
k_{m}(V)=k_{m}^{0}\left(1+\frac{r_{A} V}{V_{0}+V+r_{A} R}\right)
$$

where $r_{A}=K_{V} / K_{R}$ is the ratio between the affinities of VEGF $\left(K_{V}\right)$ and anti-VEGFR $\left(K_{R}\right)$ for the VEGFR. The metabolic stimulus equation (3) is modified similarly. It is noteworthy that $r_{A}$ is a key parameter whose influence on the system will be analysed. 

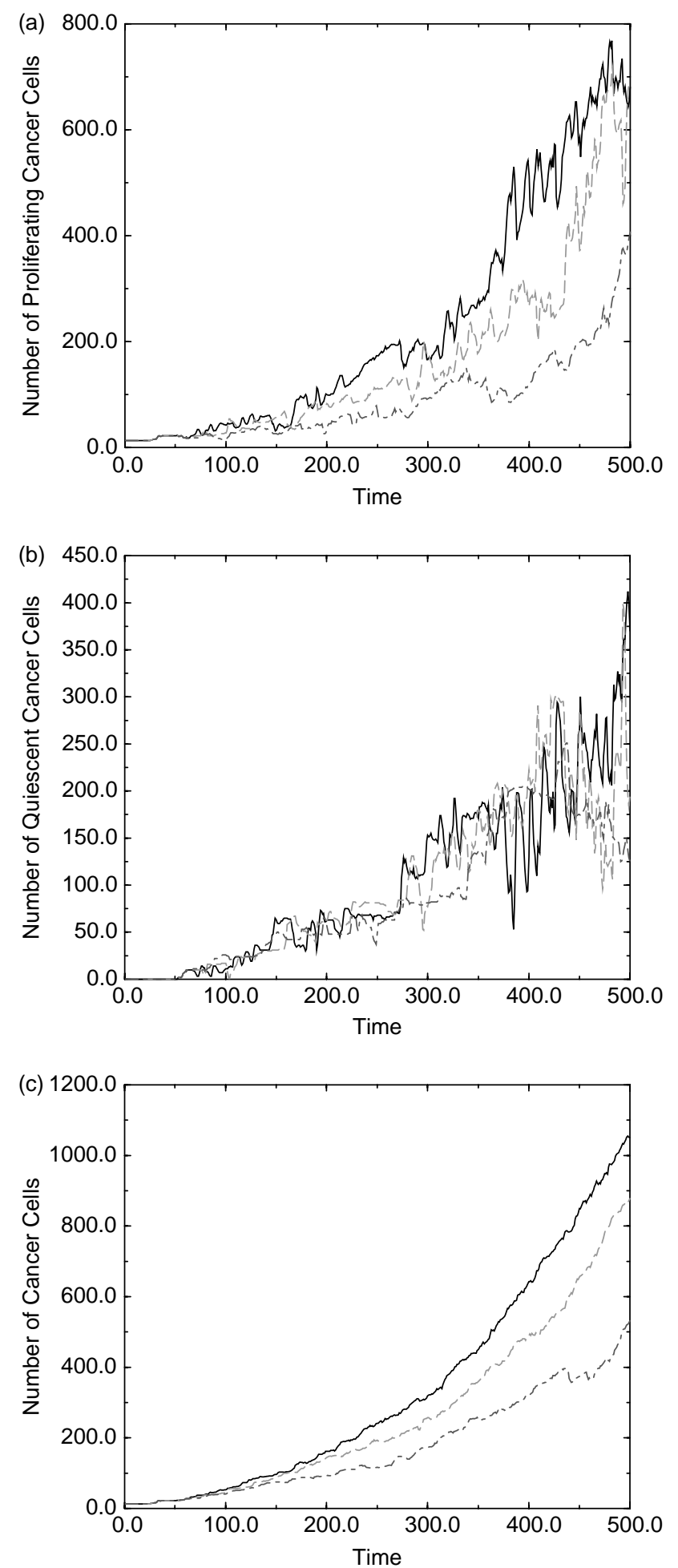


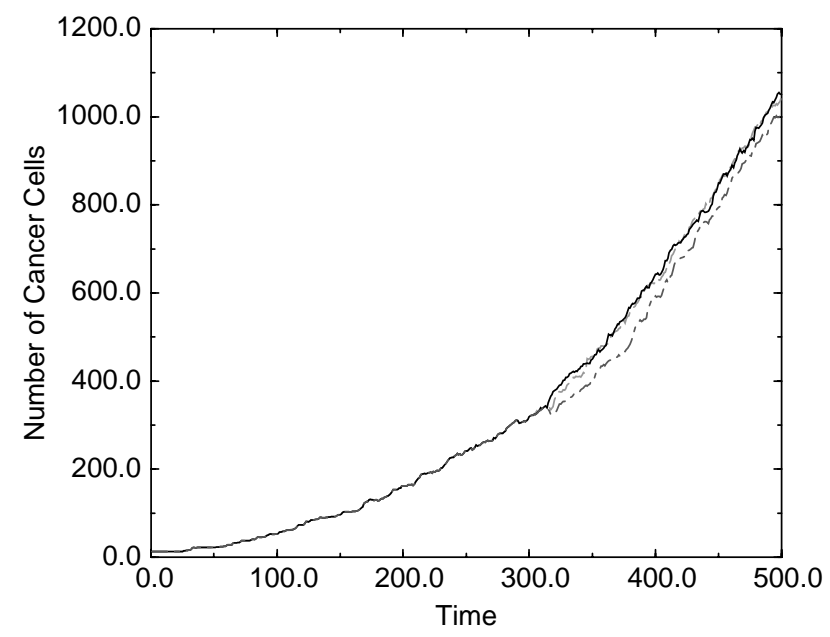

Figure 14. Time evolution of the total number of cancer cells. Black, solid lines correspond to simulations without anti-VEGFR, Red, dashed lines to simulations with anti-VEGFR $r_{A}=1$ and blue, dash-dotted lines to simulations with anti-VEGFR $r_{A}=10$. Parameter values $\eta=5 k_{m}^{0}, h_{R}=0.07$ and $D_{R}=D_{V}$. Cytotoxic drug is not included in these simulations.

\subsection{Simulation results: continuous infusion of anti-VEGFR with no cytotoxic drug}

The results in figure 13 show that continuous infusion of anti-VEGFR can reduce the size and growth rate of the tumour. It is not surprising that the larger the value of $r_{A}$ (corresponding to an increased ability of the anti-VEGFR to bind the VEGFR), the stronger is the effect of the drug on the tumour. However, the anti-VEGFR antibody does not seem able to completely eradicate the tumour and therefore as soon as the treatment is removed the tumour will regrow.

\subsection{Simulation results: bolus injection of anti-VEGFR}

Figures 14 and 15 show results where a single dose of anti-VEGFR antibody is administered between $t=300$ and $t=375$ with no cytotoxic drug. We note that a bolus injection of antiVEGFR does not have a significant effect on the size of the tumour (figure 14), although the results are slightly better for higher values of $r_{A}$. Furthermore, our model seems to be only partially successful in reproducing the normalisation window induced by anti-VEGFR in experiments [32]. To assess this, we compare the size of the quiescent (hypoxic) subpopulation upon anti-VEGFR administration with that for untreated dynamics. Figures 14(a),(b), corresponding to $r_{A}=1$, reveal that the anti-VEGFR therapy has almost no effect on the size of the quiescent subpopulation. The results corresponding to $r_{A}=10$ are slightly better: we observe a (modest) reduction in the size of the quiescent subpopulation following treatment with anti-VEGFR (approximately between $t=400$ and $t=440$, see figure $14(\mathrm{~d})$ ) (figure 16).

Figure 13. Time evolution of the number of (a) proliferating cancer cells, (b) quiescent cancer cells and (c) cancer cells. Black, solid lines correspond to simulations without anti-VEGFR, Red, dashed lines to simulations with antiVEGFR $r_{A}=1$ and blue, dash-dotted lines to simulations with anti-VEGFR $r_{A}=10$. Parameter values: $\eta=5 k_{m}^{0}$, $h_{R}=0.07$ and $D_{R}=D_{V}$. Cytotoxic drug is not included in these simulations. 

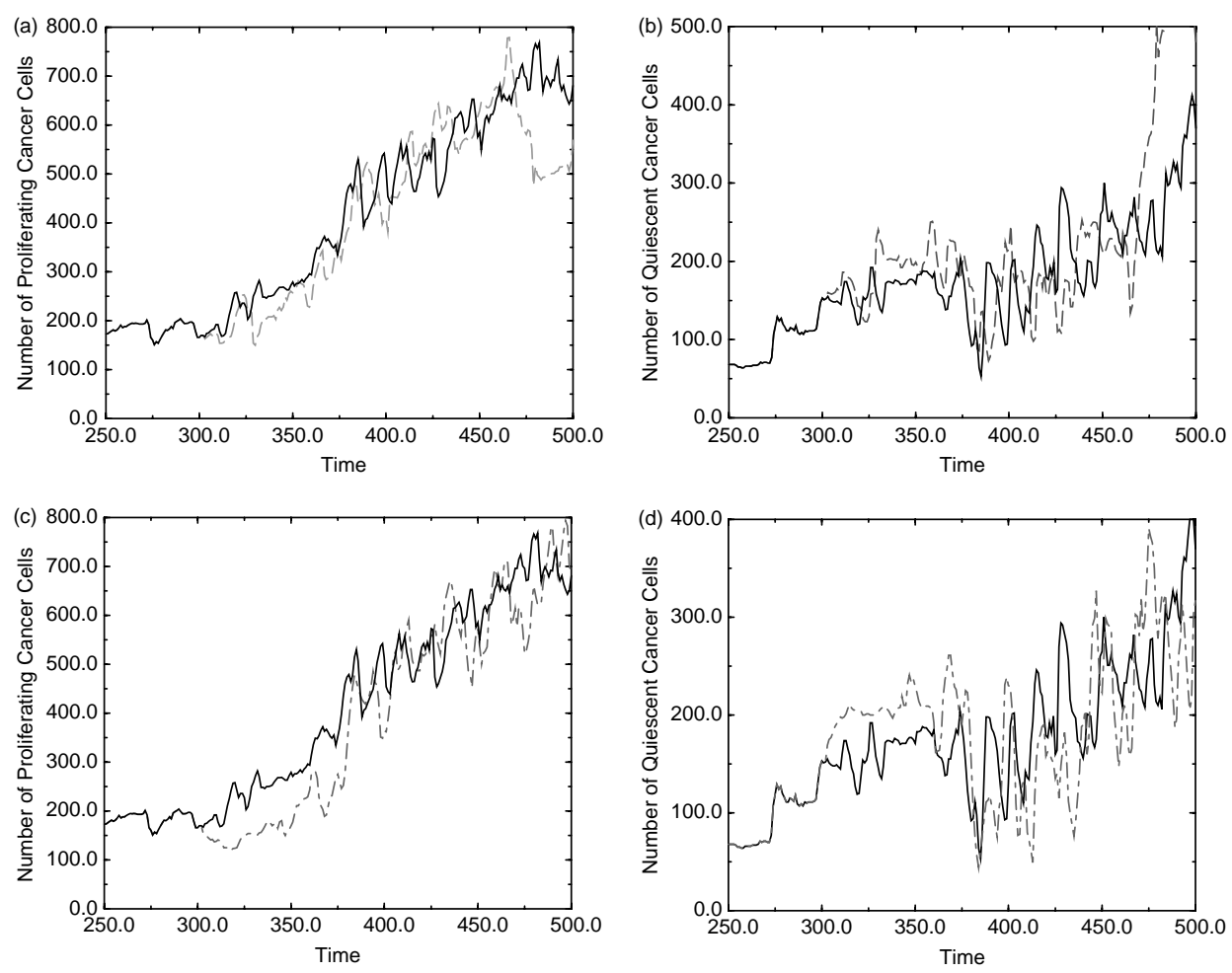

Figure 15. Time evolution of the number of proliferating and quiescent cancer cells. Panels (a) and (b) correspond to $r_{A}=1$ whereas panels (c) and (d) correspond to $r_{A}=10$. Black, solid lines correspond to simulations without anti-VEGFR, red, dashed lines to simulations with anti-VEGFR $r_{A}=1$ and blue, dash-dotted lines to simulations with anti-VEGFR $r_{A}=10$. Parameter values $\eta=5 k_{m}^{0}, h_{R}=0.07$ and $D_{R}=D_{V}$. Cytotoxic drug is not included in these simulations.

To assess the effect of the combination of anti-VEGFR and cytotoxic drug, we performed simulations in which a bolus injection of cytotoxic drug was delivered between $t=410$ and $t=415$ (Protocol 1) $\dagger$ and between $t=410$ and $t=411$ (Protocol 2) to a tumour in the presence (and absence) of anti-VEGFR therapy (the anti-VEGFR drug was administered using the protocol described above). We find that the combined therapy is more efficient than chemotherapy alone, both in terms of the percentage of cells killed, $K$, and the recovery period, as measured by the time the tumour needs to recover to a $50 \%$ of its size prior to treatment (in units of treatment duration) $T_{1 / 2}$. The results are summarised in table 1 .

If the tumour is treated with a cytotoxic drug at $t=450$ (figure 17), i.e. outside the window in which the size of the quiescent subpopulation is reduced with respect to the anti-VEGFRuntreated case (figure 14(d)), we find that the anti-VEGFR drug has virtually no effect on the response to chemotherapy. Thus, we conclude that our model exhibits normalisation-window behaviour.

$\dagger$ In fact, this combination is far from optimal as the damage it produces to the host normal tissue is massive. However, in the present study, we ignore this fact as we do not intend to carry out a thorough analysis of therapeutical protocols. Instead, this is a proof of concept exercise as to whether our model is able to capture the essential features observed in experiments. 

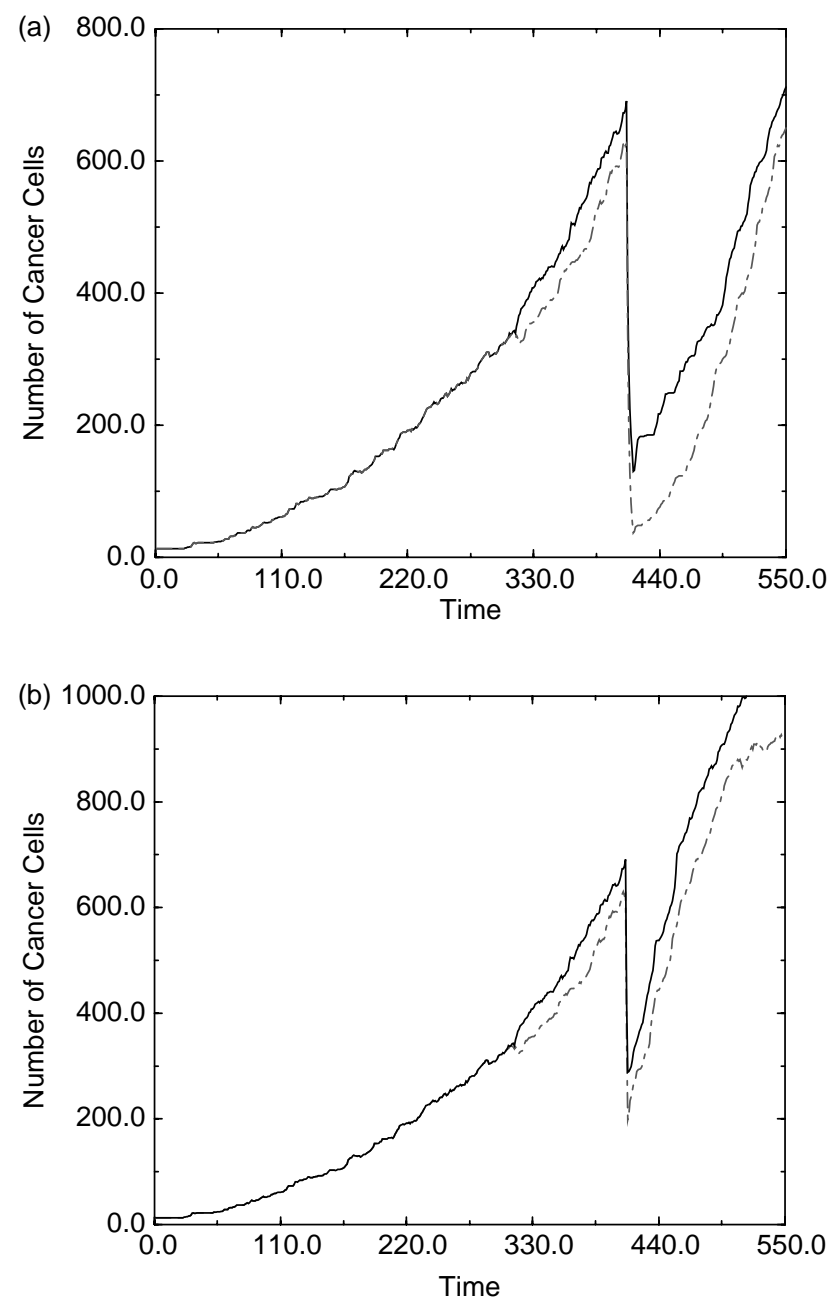

Figure 16. Time evolution of the total number of cancer cells. Black, solid lines correspond to simulations without anti-VEGFR, blue, dash-dotted lines to simulations with anti-VEGFR $r_{A}=10$. Anti-VEGFR is administered between $t=300$ and $t=375$. Panels (a) and (b) correspond to Protocol 1 and 2, respectively (see text). $\eta=5 k_{m}^{0}$, $h_{R}=0.07 . D_{R}$ has been taken to have the same value as $D_{V}$.

We have run simulations with $V_{\mathrm{c}}=0.001$ and $V_{\mathrm{c}}=0.01$, i.e. 2 and 20 times bigger than in the base-case scenario, where we take $V_{\mathrm{c}}=0.0005$. In figure 18 we can see that when $V_{\mathrm{c}}=$ 0.001 the window during which the size of the quiescent subpopulation is reduced decreases with respect to the case with $V_{\mathrm{c}}=0.0005$, and it is further reduced for $V_{\mathrm{c}}=0.01$. There is also a consistent tendency to delay the appearance of the window when $V_{\mathrm{c}}$ is increased (figure 19).

Table 1. Summary of the results obtained using the different protocols described in Section 7.3.

\begin{tabular}{lcccc}
\hline & Protocol 1 & Protocol 1 with anti-VEGFR & Protocol 2 & Protocol 2 with anti-VEGFR \\
\hline$K$ & $80 \%$ & $90 \%$ & $53 \%$ & $67 \%$ \\
$T_{1 / 2}$ & 13 & 16 & 5 & 10 \\
\hline
\end{tabular}




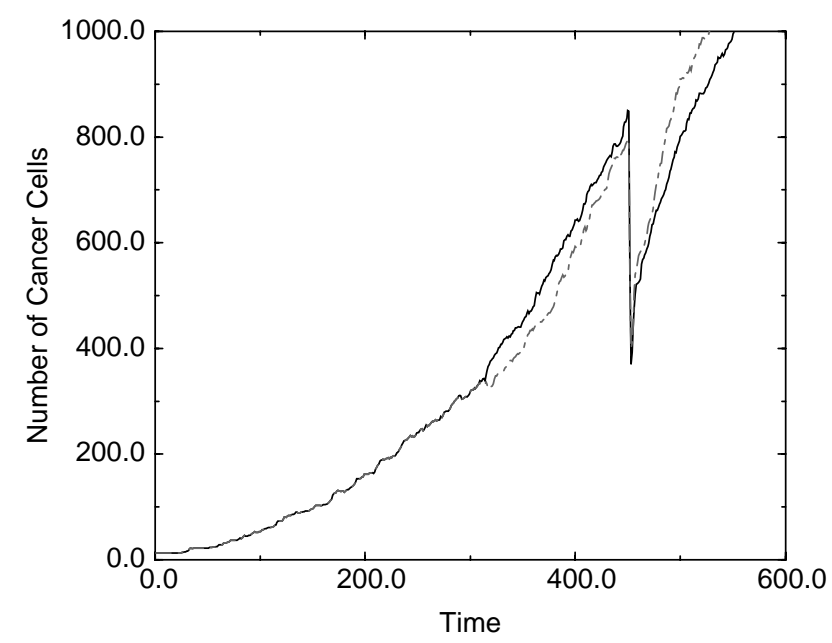

Figure 17. Time evolution of the total number of cancer cells. Black, solid lines correspond to simulations without anti-VEGFR, blue, dash-dotted lines to simulations with anti-VEGFR $r_{A}=10$. Cytotoxic drug administered at 450 . Anti-VEGFR is administered between $t=300$ and $t=375 . \eta=5 k_{m}^{0}, h_{R}=0.07 . D_{R}$ has been taken to have the same value as $D_{V}$.

In view of these results, we conclude that our model is consistent with recent findings regarding the combination of antiangiogenic therapy with cytotoxic drugs and radiotherapy, although it predicts only minor improvement in cytotoxic drug efficacy. There are several reasons for this under-estimation of the efficacy of combined therapy. One of them is very likely to be the fact that angiogenesis is treated implicitly through the VEGF-dependent structural adaptation. However, there are other possibilities, one being related to our model of vessel co-option and dematuration. Upon co-option, vessel behaviour is determined by the level of VEGF: low levels of VEGF lead to vessel collapse whereas high levels yield angiogenic vessels. In our model, the transition from one type of behaviour to the other is determined in terms of the level of VEGF compared to a threshold value, $V_{\mathrm{c}}$. In the presence of an anti-VEGFR drug, it is feasible that higher levels of VEGF are needed to trigger angiogenic behaviour or, equivalently, the threshold $V_{\mathrm{c}}$ becomes larger in the presence of anti-VEGFR. Detailed modelling of the dependence of $V_{c}$ on VEGF concentration is beyond the scope of the present work. Instead, we run simulations with increased values of $V_{\mathrm{c}}$ for the duration of the anti-VEGFR treatment to assess the effect that this parameter has on the behaviour of the system.

From figure 15 we observe that, although $V_{\mathrm{c}}$ has an effect on the number of cancer cells killed by the anti-VEGFR drug alone, it does not seem to have much influence on the outcome of the combined therapy in terms of its effect on tumour size.

\section{Discussion}

In this paper, we have presented a multiscale model for vascular tumour growth and tumourinduced vessel dematuration. We have used the model to analyse the effect of combined antiangiogenic and cytotoxic therapies.

Upon co-option by a growing tumour mass, blood vessels undergo a process of dematuration, which initially, in the absence of VEGF, induces vessel collapse. This, in turn, 

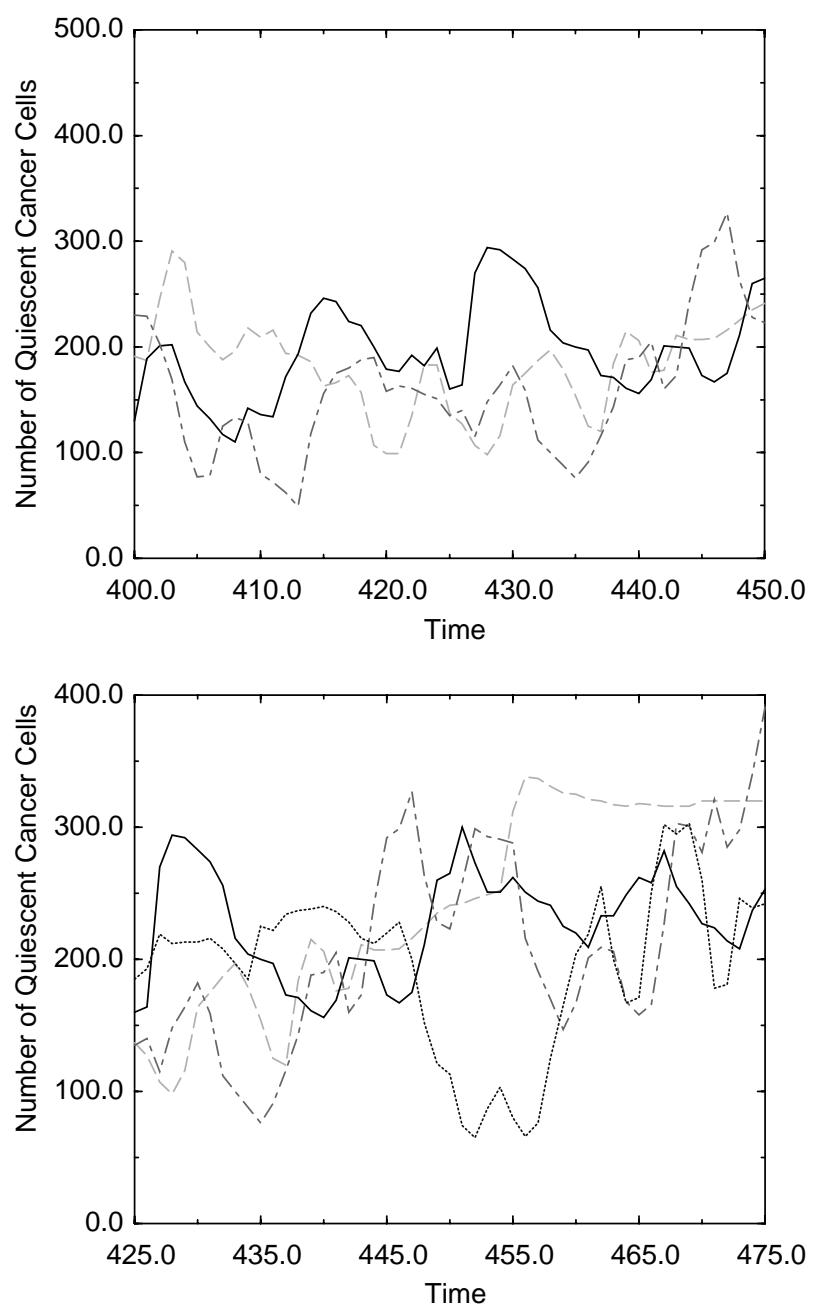

Figure 18. Time evolution of the number of quiescent cancer cells. Black, solid lines correspond to simulations without anti-VEGFR, blue, dash-dotted lines to simulations with anti-VEGFR with $V_{\mathrm{c}}=0.0005$, green, dashed lines correspond to simulations with anti-VEGFR with $V_{\mathrm{c}}=0.001$, and brown, dotted lines correspond to simulations with anti-VEGFR with $V_{\mathrm{c}}=0.01 . r_{A}=10$. Anti-VEGFR is administered between $t=300$ and $t=375 . \eta=5 k_{m}^{0}$, $h_{R}=0.07 . D_{R}$ has been taken to have the same value as $D_{V}$. No cytotoxic drug is present in these simulations.

creates large hypoxic regions which render the tumour more resistant to cytotoxic drugs. Furthermore, when cells are killed by the cytotoxic drug, the available oxygen increases, enabling quiescent cells to re-enter the cell-cycle and thereby allowing the tumour to progress. Thus, the results presented in section 6 suggest a more complex scenario than that proposed by Teicher [29], in which a combination of antiangiogenic and cytotoxic therapies is more efficient than each one on its own because two cancer compartments are targeted. Our model suggests a subtle interplay between the dynamics of tumour subpopulations, oxygen supply and vascular dynamics.

In section 7, we introduced into our model an antiangiogenic drug, in particular an antibody against the VEGF receptor, which inhibits the effect of VEGF by competitive 


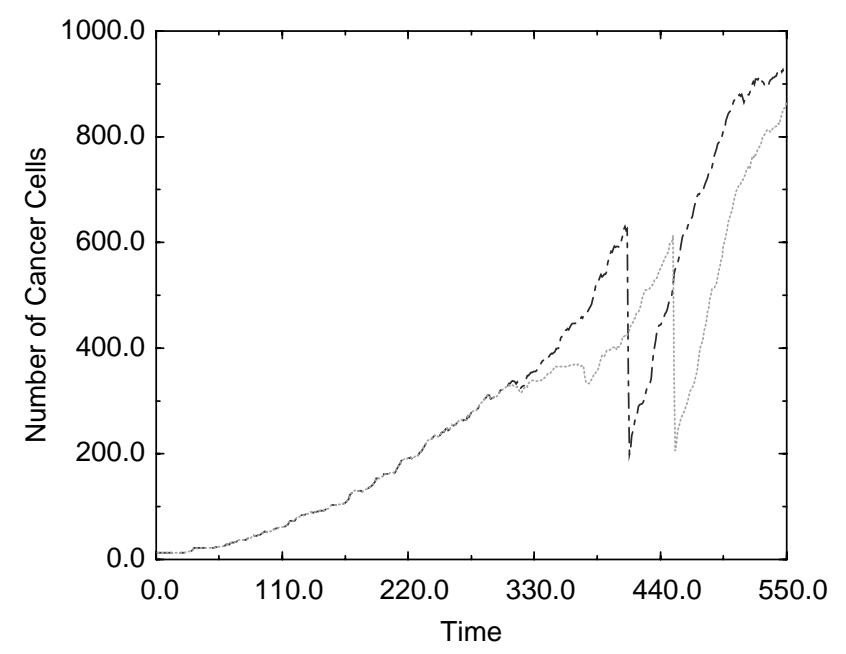

Figure 19. Time evolution of the total number of cancer cells. Blue, dash-dotted lines correspond to simulations with anti-VEGFR with $V_{\mathrm{c}}=0.0005$ and cytotoxic drug administered at $t=410$. Brown, dotted lines correspond to simulations with anti-VEGFR with $V_{\mathrm{c}}=0.01$ and cytotoxic drug administered at $t=450 . r_{A}=10$. Anti-VEGFR is administered between $t=300$ and $t=375 . \eta=5 k_{m}^{0}, h_{R}=0.07$. $D_{R}$ has been taken to have the same value as $D_{V}$.

binding to the VEGFR. This type of therapy has been used in experiments in combination with radio- and chemo-therapy [30,32]. Our model simulations are consistent with these reproducing two basic experimental (and clinically-supported) features, namely, the improved efficacy of cytotoxic drugs following treatment with anti-VEGFR drug, and the emergence of a window of opportunity, i.e. period of time following treatment with antiVEGFR during which such improvement is possible. Administration of a cytotoxic drug before or after this window does not improve its impact. The mechanism by which our model produces better therapeutic outcomes when therapies are combined is related to a reduction in the size of the quiescent subpopulation, caused by enhanced tumour oxygenation. This mechanism is also consistent with experimental observations [32].

The mechanism by which our model produces the window of opportunity is due to the interplay between vascular structural adaptation, oxygen supply and the dynamics of the subpopulations of quiescent and proliferating cells. The presence of the anti-VEGFR drug produces extensive hypoxic regions within the tumour, which leads, on the one hand, to massive VEGF production and, on the other, to a certain amount of cell death by oxygen starvation, the extent of which depends, among other factors, on the value of the critical VEGF concentration for collapsing-to-angiogenic transition in an engulfed vessel $\left(V_{\mathrm{c}}\right)$. The great amounts of VEGF produced stimulates reorganisation of the vasculature to bring oxygen to the hypoxic regions. This effect, together with the reduction in oxygen demand caused by cell death, leads to reoxygenation of hypoxic regions and a temporary reduction in the size of the quiescent subpopulation. Eventually, other regions of the tumour become hypoxic and blood flow undergoes another period of spatial reorganisation and the window of opportunity closes.

In spite of good qualitative agreement between our simulations and experimental observations, there are aspects of the model that need to be improved. The mechanism described above explaining the appearance of a window of opportunity is feasible but it is unlikely to be the only mechanism at work. For example, angiogenesis and vascular 
remodelling by vessel pruning are likely to be involved. Inclusion of these processes will be necessary to gain a more thorough understanding of the phenomenon under study.

Another limitation of our multiscale model concerns its numerical implementation. In particular, the diffusion of chemicals and structural adaptation are supposed to occur on a much shorter timescale than cell division and all the processes involved in our multiscale model are assumed to be slaved to the intracellular dynamics. Whilst this assumption is supported by experimental data, in order to produce accurate predictions for quantities such as the duration of the normalisation window and the waiting time between the end of the antiangiogenic therapy and the beginning of the normalisation window, both of major importance for clinical applications, we most likely need to account for the timescales of VEGF clearance, vascular adaptation, angiogenesis and vessel pruning. This not only requires a thorough re-implementation of the model, but is also related to fundamental questions regarding numerical implementation of multiscale models such as how to achieve a time stepping scheme that is both efficient numerically and that produces realistic results. These model drawbacks will be the subject of future research.

\section{Acknowledgements}

TA would like to thank the EPSRC for financial support under grant GR/509067.

\section{References}

[1] Alarcón, T., Byrne, H.M. and Maini, P.K., 2003, A cellular automaton model for tumour growth in a heterogeneous environment, Journal of Theoretical Biology, 225, 257-274.

[2] Alarcón, T., Byrne, H.M. and Maini, P.K., 2004, A mathematical model of the effects of hypoxia on the cellcycle of normal and cancer cells, Journal of Theoretical Biology, 229, 395-411.

[3] Alarcón, T., Byrne, H.M. and Maini, P.K., 2005, A multiple scale model of tumour growth, Multiscale Modeling and Simulation, 3, 440-475.

[4] Bartha, K. and Rieger, H., 2006, Vascular network remodelling via vessel co-option, regression and growth in tumours, Preprint. Available from the arXiv/Q-Bio repository: http://arxiv.org/abs/q-bio.TO/0506039.

[5] Byrne, H.M., Owen, M.R., Alarcón, T., Murphy, J. and Maini, P.K., 2006, Modelling the response of vascular tumours to chemotherapy: a multiscale approach, Mathematical Models and Methods in Applied Sciences, 16(7S), 1-25.

[6] Deutsch, A. and Dormann, S., 2002, Modeling of a vascular tumor growth with a hybrid cellular automaton, In Silico Biology, 2, 1-14.

[7] Gardner, L.B., Li, Q., Parks, M.S., Flanagan, W.M., Semenza, G.L. and Dang, C.V., 2001, Hypoxia inhibits $\mathrm{G}_{1} / \mathrm{S}$ transition through regulation of $\mathrm{p} 27$ expression, The Journal of Biological Chemistry, 276, 7919-7926.

[8] Gatenby, R.A. and Gawlinski, E.T., 2003, The glycolytic phenotype in carcinogenesis and tumor invasion: insights through mathematical models, Cancer Research, 63, 3847-3854.

[9] Gatenby, R.A. and Maini, P.K., 2004, Mathematical oncology, Nature, 421, 321.

[10] Green, S.L., Freiberg, R.A. and Giaccia, A., 2001, p $21^{\mathrm{Cip} 1}$ and $\mathrm{p} 27^{\mathrm{Kip} 1}$ regulate cell cycle reentry after hypoxic stress but are not necessary for hypoxia-induced arrest, Molecular and Cellular Biology, 21, 1196-1206.

[11] Hunter, P.J., Robbins, P. and Noble, D., 2002, The IUPS human physiome project, Pflügers Archiv-European Journal of Physiology, 445, 1-9.

[12] Jain, R.K., 2001, Delivery of molecular and cellular medicine to solid tumours, Advanced Drug Delivery Reviews, 46, 149-168.

[13] Jain, R.K., 2003, Molecular regulation of vessel maturation, Nature Medicine, 9, 685-693.

[14] Jain, R.K., 2005, Normalization of tumour vasculature: an emerging concept in antiangiogenic therapy, Science, 307, 58-62.

[15] Keener, J. and Sneyd, J., 1998, Mathematical Physiology (New York, NY (USA): Springer-Verlag).

[16] Maini, P.K., Alarcón, T., Byrne, H.M., Owen, M.R. and Murphy, J., 2000, To appear Structural adaptation in normal and cancerous vasculature. In: G. Aletti, M. Burger, A. Micheletti and D. Morale (Eds.) Math Everywhere. A Volume Dedicated to Vincenzo Capasso's 60th Birthday.

[17] Mantzaris, N.V., Webb, S. and Othmer, H.G., 2004, Mathematical modelling of tumour-induced angiogenesis, Journal of Mathematical Biology, 49, 111-187. 
[18] Mayer, R.J., 2004, Two steps forward in the treatment of colorectal cancer, The New England Journal of Medicine, 350, 2406-2408.

[19] McDougall, S.R., Anderson, A.R.A. and Chaplain, M.A.J., Mathematical modelling of dynamic adaptive tumour-induced angiogenesis: clinical implications and therapeutic targeting strategies, Journal of Theoretical Biology, In press.

[20] Moreira, J. and Deutsch, A., 2002, Cellular automaton models of tumor development: a critical review, Advances in Complex Systems, 5, 247-267.

[21] Patel, A.A., Gawlinski, E.T., Lemieux, S.K. and Gatenby, R.A., 2001, A cellular automaton model of early tumor growth and invasion: the effects of native tissue vascularity and increased anaerobic tumor metabolism, Journal of Theoretical Biology, 213, 315-331.

[22] Philipp-Staheli, J., Payne, S.R. and Kemp, C.J., 2001, p27(Kip1): regulation and function of haploinsufficient tumour suppressor and its misregulation in cancer, Experimental Cell Research, 264, 148-168.

[23] Pries, A.R., Secomb, T.W. and Gaehtgens, P., 1998, Structural adaptation and stability of microvascular networks: theory and simulations, The American Journal of Physiology, 275, H349-H360.

[24] Pries, A.R., Reglin, B. and Secomb, T.W., 2001, Structural adaptation of microvascular networks: functional response to adaptive responses, The American Journal of Physiology, 281, H1015-H1025.

[25] Ribba, B., Marron, K., Agur, Z., Alarcón, T. and Maini, P.K., 2005, A mathematical model of doxorubicin treatment efficacy on non-Hodgkin lymphoma: investigation of current protocol through theoretical modelling results, Bulletin Mathematical Biology, 67, 79-99.

[26] Resnick, N., Yahav, H., Shay-Salit, A., Shushy, M., Schubert, S., Zilberman, L.C.M. and Wofovitz, E., 2003, Fluid shear stress and the vascular endothelium: for better and for worse, Progress in Biophysics and Molecular Biology, 81, 177-199.

[27] Royds, J.A., Dower, S.K., Qwarstrom, E.E. and Lewis, C.E., 1998, Response of tumour cells to hypoxia: role of p53 and $\mathrm{NF}_{\mathrm{K} \beta}$, Journal of Clinical Pathology: Molecular Pathology, 51, 55-61.

[28] Stéphanou, A., McDougall, S.R., Anderson, A.R.A. and Chaplain, M.A.J., Mathematical modelling of the influence of blood rheological properties upon adaptive tumour-induced angiogenesis, Mathematical and Computer Modelling, In press.

[29] Teicher, B.A., 1996, A systems approach to cancer therapy, Cancer Metastasis Reviews, 15, $247-272$.

[30] Tong, R.T., Boucher, Y., Kozin, S.V., Winkler, F., Kicklin, D.J. and Jain, R.K., 2004, Vascular normalisation by VEGFR2 blockade induces a pressure gradient accross the vasculature and improves drug penetration tumours, Cancer Research, 64, 3731-3736.

[31] Tyson, J.J. and Novak, B., 2001, Regulation of the eukariotic cell-cycle: molecular anatagonism, hysteresis, and irreversible transitions, Journal of Theoretical Biology, 210, 249-263.

[32] Winkler, F., Kozin, S.V., Tong, R.T., Chae, S-S., Booth, M.F., Garkavtsev, I., Xu, L., Hicklin, D.J., Fukumura, D., di Tomaso, E., Munn, L.L. and Jain, R.K., 2004, Kinetics of vascular normalisation by VEGFR2 blockade governs brain tumour response to radiation: role of oxygenation, angiopoietin-1 and matrix metalloproteinases, Cancer Cell, 6, 553-563.

[33] Yancopoulos, G.D., Davis, S., Gale, N.W., Rudge, J.S., Wiegand, S.J. and Holash, J., 2000, Vascular-specific growth factors and blood vessel formation, Nature, 407, 242-248.

\section{A Mathematical description of the cellular and intracellular layers of our multiscale model}

\section{A.1 The diffusible chemicals (macroscale)}

Coupling between the vascular, cellular and subcellular scales is effected by the diffusive transport of oxygen and VEGF, under the assumption that oxygen is the single, growth-rate limiting nutrient. We calculate their respective distributions within the tissue by solving appropriate reaction-diffusion equations and imposing zero-flux boundary conditions. We justify adopting the usual quasi-steady approximation in these equations on the grounds that the timescales for oxygen and VEGF diffusion are much shorter than the tumour doubling time which is the timescale of interest (minutes and weeks or months, respectively).

We denote by $P$ the oxygen concentration and treat the cells as sinks and the vessels as sources of oxygen so that the relevant diffusion equation can be written

$$
0=D_{P} \nabla^{2} P+h_{P}\left(P_{\text {vess }}-P\right)-\lambda_{\text {cell }} P .
$$


In equation (A1), $D_{P}$ denotes the assumed constant oxygen diffusion coefficient, $h_{P}$ is the rate at which oxygen is transported across the vessel wall $\left(h_{P}\right.$ is only non-zero where vessels are located), $P_{\text {vess }}=P_{\text {vess }}(H)$ is the oxygen concentration associated with a haematocrit $H$ (this is determined from the vascular problem). Finally, $\lambda_{\text {cell }}$ denotes the assumed constant rate at which cells consume oxygen, with different values for normal and cancerous cells.

The local VEGF profile is calculated in a similar manner, except that cells now act as sources of VEGF and vessels as sinks. If we further assume that VEGF is rapidly eliminated from the vasculature (so that its concentration there is zero) and denote by $\lambda_{V}$ its natural halflife, then $V$ satisfies

$$
0=D_{V} \nabla^{2} V-h_{V} V+\gamma_{\text {cell }}-\lambda_{V} V .
$$

In equation (A2), $D_{V}$ denotes the assumed constant diffusion coefficient of VEGF and $h_{V}$ the rate at which VEGF binds the EC surface at the vessel walls. Finally, $\gamma_{\text {cell }}$ represents the rate at which sites occupied by cells release their intracellular stores of VEGF to the extracellular environment (this occurs only when the internal levels of VEGF exceed a threshold value). As with $\lambda_{\text {cell }}$ in equation (A1), $\gamma_{\text {cell }}$ differs between normal and cancerous cells.

We note that equations (A1) and (A2) for $P$ and $V$ above differ from those used in Ref. [3]. There the vessels were treated as boundaries and exchange with the vasculature was incorporated as an internal boundary condition rather than a distributed source term. With a hexagonal network of vessels, this meant that oxygen and VEGF were confined to hexagonal regions of the tissue.

\section{A.2 The cellular level}

The dynamics of the cell colony is modelled using a two-dimensional cellular automaton, with $N \times N$ automaton elements or cells [20]. Each element is characterised by a state vector, whose components correspond to features of interest. These include: (i) occupation status (whether an element is occupied by a normal cell, a cancer cell, an empty space or a vessel), (ii) cell status (whether the cell is in a proliferative or quiescent state), (iii) the local oxygen concentration, and (iv) the intracellular levels of VEGF, p53 and cell-cycle proteins (see section 2.4). The state vector evolves according to prescribed local rules that update a given element on the basis of its own state and those of its neighbours at the previous time step [1]. These rules were inspired by generic features of tumour growth, such as the ability of cancer cells to elude the control mechanisms which maintain stasis in normal tissues. They can also manipulate their local environment, providing themselves with better conditions for growth and, eventually, for invasion of the host organism [8]. Additionally, we endow the cancer cells with the ability to survive exposure to hypoxia for longer than their normal counterparts.

For each time step, elements of the cellular automaton are selected randomly and updated in turn. If a particular element is occupied by a cell, we update its internal dynamics (cell cycle proteins, VEGF and p53 levels). If a cell is ready to divide, the daughter cell is placed in the adjacent cell with the largest oxygen concentration conditions. If there is no empty site, the cell fails to divide and dies. The mechanism for cell death or apoptosis differs between normal and cancer cells. In normal cells, if the intracellular level of p53 exceeds a threshold value (which depends on the cellular composition of its nearest neighbours), then the cell dies. By contrast, under low oxygen, cancer cells become quiescent, suspending most cell functions, including proliferation. On entering this state, a clock is started. It is incremented by unit steps for each time step that the cell remains quiescent. If the clock reaches a 
threshold value then the cell dies. However, if the oxygen level increases above the threshold for cancer cell quiescence then the cell recommences cycling and its clock is reset to zero.

\section{A.3 The subcellular level}

We formulate systems of ODEs to describe the evolution of the chemicals that control the subcellular processes of interest. These include progress through the cell cycle, VEGF and p53 expression.

The cell cycle is regulated by complex interactions between a large number of proteins, the key components being the families of cyclin-dependent kinases (CDKs) and the cyclins. The activity of cyc-CDK complexes is low during G1 and becomes high after transition. In addition, activities of the anaphase protein complex (APC) and the protein Cdh1 are such that their levels are high in G1 but become low after the G1/S transition. For simplicity, we base our work on the model of Tyson and Novak [31], which captures the essential features of the cell cycle and can be written in the following form:

$$
\begin{gathered}
\frac{\mathrm{d} x}{\mathrm{~d} t}=\frac{\left(k_{3}^{\prime}+k_{3}^{\prime \prime} A\right)(1-x)}{J_{3}+1-x}-\frac{k_{4} m y x}{J_{4}+x}, \\
\frac{\mathrm{d} y}{\mathrm{~d} t}=k_{1}-\left(k_{2}^{\prime}+k_{2}^{\prime \prime} x\right) y, \\
\frac{\mathrm{d} m}{\mathrm{~d} t}=\mu m\left(1-\frac{m}{m_{*}}\right)
\end{gathered}
$$

where $x \equiv[\mathrm{Cdh} 1]$ is the concentration of active Cdh1/APC complexes, $y \equiv[\mathrm{Cyc}]$ is the concentration of cyclin-CDK complexes and $m$ is the mass of the cell. The parameters $k_{i}$ $(i=1,2,3,4)$ and $J_{i}(i=3,4)$ are positive constants and $A$ represents a generic activator. In equation (A5), $\mu$ is the cell growth rate and $m_{*}$ is the mass of an adult cell.

Equations (A3)-(A5) can exhibit mono- and bi-stability, with the cell mass $m$ as a bifurcation parameter. For low values of $m$ there is a single stable steady state with a high value of $x$ and a low value of $y$-this corresponds to G1. As $m$ increases, the system becomes bistable and a new stable steady state characterised by a high value of $y$ and a low value of $x$ emerges. For a critical value of $m$ the latter becomes the only stable steady state and the system switches to this state, corresponding to the $\mathrm{S}$ phase. After the cell divides, $m$ decreases, and the system is re-set to the "G1 phase steady state".

Guided by experimental results presented in Ref. [7] and the hypothesis that under hypoxia expression of the regulatory protein p27 increases [10], we generalise equations (A3)-(A5) and study the (non-dimensionalised) model (see [2] for full details):

$$
\begin{gathered}
\frac{\mathrm{d} x}{\mathrm{~d} \tau}=\frac{\left(1+b_{3} u\right)(1-x)}{J_{3}+1-x}-\frac{b_{4} m x y}{J_{4}+x}, \\
\frac{\mathrm{d} y}{\mathrm{~d} \tau}=a_{4}-\left(a_{1}+a_{2} x+a_{3} z\right) y,
\end{gathered}
$$




$$
\begin{gathered}
\frac{\mathrm{d} m}{\mathrm{~d} \tau}=\eta m\left(1-\frac{m}{m_{*}}\right), \\
\frac{\mathrm{d} z}{\mathrm{~d} \tau}=\chi(m)-c_{2} \frac{P}{B+P} z, \\
\frac{\mathrm{d} u}{\mathrm{~d} \tau}=d_{1}-\left(d_{2}+d_{1} y\right) u,
\end{gathered}
$$

where now $P$ is the oxygen tension (see equation (A1)), $z$ is the p27 concentration and $u$ is the concentration of phosphorylated retinoblastoma (RB). In equations (A6)-(A10), we assume that normal and cancer cells differ only in the rates at which they produce p27. For normal cells, p27 expression is regulated by cell size and we fix $\chi(m)=c_{1}^{n}\left(1-\left(\mathrm{m} / \mathrm{m}_{*}\right)\right)$. For cancerous cells, this size-regulation is assumed lost and $\chi(m)=c_{1}^{t}$. To account for the lower levels of p27 observed in cancer cells compared to normal cells [22] we further assume that the maximum rate of $\mathrm{p} 27$ synthesis in normal cells exceeds that in cancer cells (i.e. $\left.c_{1}^{n}>c_{1}^{t}\right)$.

Equation (A6) renders explicit how our cell cycle model is coupled to the tissue level by the oxygen concentration. As we now explain, the other intracellular processes of interest (i.e. VEGF production and apoptosis) are also influenced by $P$. In normal and cancer cells hypoxia stimulates the expression of VEGF and the protein p53. In normal cells p53 stimulates apoptosis and may inhibit VEGF production [27]. In cancer cells mutations in p53 are common and the protein may upregulate VEGF production and/or prevent apoptosis.

We denote by $p$ and $q$, respectively, the concentrations of p53 and VEGF within a given cell and assume that their evolution is modulated by $P$ in the following manner:

$$
\begin{gathered}
\frac{\mathrm{d} p}{\mathrm{~d} t}=k_{7}-\frac{k_{7}^{\prime} P}{C+P} p, \\
\frac{\mathrm{d} q}{\mathrm{~d} t}=\xi(p, q)-\frac{k_{8}^{\prime} P}{D+P} q,
\end{gathered}
$$

where

$$
\xi(p, q)= \begin{cases}k_{8}-k_{8}^{n} \frac{p q}{J_{s}^{n}+q} & \text { for normal cells } \\ k_{8}+k_{8}^{t} \frac{p q}{J_{s}^{t}+q} & \text { for cancer cells }\end{cases}
$$

and all parameters are positive. Thus VEGF expression increases under hypoxia, and p53 increases VEGF production in cancer cells and decreases it in normal cells. The intracellular levels of $p$ and $q$ are assigned to the automaton state vector. For details on the experimental evidence supporting the forms of equations (A11) and (A12) the reader is referred to our previous work [3]. 
Table A1. Parameter values corresponding to the new quantities introduced in the present work.

\begin{tabular}{lll}
\hline Parameter & \multicolumn{1}{c}{ Value } & Units \\
\hline$D_{R}$ & $2.41 \times 10^{-5}$ & $\mathrm{~cm}^{2} / \mathrm{seg}$ \\
$\lambda_{R}$ & 5 & $1 / \mathrm{min}$ \\
$h_{R}$ & 0.07 & $1 / \mathrm{min}$ \\
$D_{\theta}$ & $2.41 \times 10^{-5}$ & $\mathrm{~cm}^{2} / \mathrm{seg}$ \\
$\lambda_{\theta}$ & 2 & $1 / \mathrm{min}$ \\
$h_{\theta}$ & 0.07 & $1 / \mathrm{min}$ \\
$J_{0}$ & 27.9 & - \\
$\tau_{r}$ & 0.103 & $\mathrm{dyn} / \mathrm{cm}^{2}$ \\
$k_{p}$ & 0.68 & - \\
\hline
\end{tabular}

Table A2. Parameter values used for our cell cycle, VEGF secretion and apoptosis models.

\begin{tabular}{lccc}
\hline Parameter & Value (normal) & Value (cancer) & Units \\
\hline$k_{1}$ & 0.04 & 0.04 & $\min ^{-1}$ \\
$k_{2}^{\prime}$ & 0.5 & 0.4 & $\min ^{-1}$ \\
$k_{2}^{\prime \prime}$ & 1 & 1 & $\min ^{-1}$ \\
$k_{2}^{\prime \prime \prime}$ & 0.25 & 0.25 & $\min ^{-1}$ \\
$k_{3}^{\prime}$ & 1 & 1 & $\min ^{-1}$ \\
$k_{3}^{\prime \prime}$ & 10 & 10 & $\min ^{-1}$ \\
$k_{4}^{\prime}$ & 35 & 35 & $\min ^{-1}$ \\
$\mu$ & 0.01 & 0.01 & $\min ^{-1}$ \\
$m_{*}$ & 10 & 10 & None \\
$J_{3}, J_{4}$ & 0.04 & 0.04 & one $^{-1}$ \\
$k_{5}$ & 0.1 & 0.002 & $\min ^{-1}$ \\
$k_{5}^{\prime}$ & 0.01 & 0.01 & $\min ^{-1}$ \\
$B$ & 0.01 & 0.01 & None \\
$k_{6}$ & 0.01 & 0.01 & $\min ^{-1}$ \\
$k_{6}^{\prime}$ & 0.1 & 0.1 & $\min ^{-1}$ \\
$k_{7}$ & 0.002 & 0.002 & $\min ^{-1}$ \\
$k_{7}^{\prime}$ & 0.01 & 0.01 & $\min ^{-1}$ \\
$C$ & 0.01 & 0.01 & None \\
$k_{8}$ & 0.002 & 0.002 & $\min ^{-1}$ \\
$k_{8}^{\prime}$ & 0.01 & 0.01 & $\min ^{-1}$ \\
$k_{8}^{\prime \prime}$ & 0.002 & 0.002 & $\min ^{-1}$ \\
$D$ & 0.01 & 0.01 & None \\
$J_{5}$ & 0.04 & 0.04 & None \\
\hline & & &
\end{tabular}

Table A3. Parameter values used in our simulations.

\begin{tabular}{|c|c|c|}
\hline Parameter & Value & Units \\
\hline$k_{m}$ & 0.83 & $\mathrm{~s}^{-1}$ \\
\hline$Q_{\mathrm{ref}}$ & 40 & $\mathrm{nl} / \mathrm{min}$ \\
\hline$k_{s}$ & 1.79 & $\mathrm{~s}^{-1}$ \\
\hline$\alpha$ & 0.5 & None \\
\hline THR & 2.5 & None \\
\hline$D_{P}$ & $2.41 \times 10^{-5}$ & $\mathrm{~cm}^{2} \mathrm{~s}^{-1}$ \\
\hline$K_{N}$ & $1.57 \times 10^{-4}$ & $m l O_{2} \mathrm{ml}^{-1} \mathrm{~s}^{-1}$ \\
\hline$K_{T}$ & $1.57 \times 10^{-4}$ & $\mathrm{mlO}_{2} \mathrm{ml}^{-1} \mathrm{~s}^{-1}$ \\
\hline$h_{P}$ & $3.0 \times 10^{-4}$ & $\mathrm{~cm} \mathrm{~s}^{-1}$ \\
\hline$q_{\mathrm{THR} 1}($ normal cells only) & 1.5 & None \\
\hline$q_{\mathrm{THR} 2}$ (normal cells only) & 2 & None \\
\hline$p_{\text {THR } 1}$ (normal cells only) & 0.08 & None \\
\hline$p_{\text {THR } 2}$ (normal cells only) & 0.8 & None \\
\hline$z_{\text {THR } 1}$ (cancer cells only) & 0.8 & None \\
\hline$z_{\mathrm{THR} 2}($ cancer cells only) & 0.8 & None \\
\hline
\end{tabular}




\section{A.4 Chemotherapy}

We now investigate how the dynamics of the normal and cancer cells change when a chemotherapeutic drug is introduced [5]. The calculation of drug concentration $\theta_{\text {vess }}$ within the vascular network is strongly related to the computation of the haematocrit, $H$ (see Section 2.1). In particular, we assume that the drug is soluble in plasma and, therefore, dissolved within the liquid fraction of blood, i.e. $1-H$. In particular, we will assume that the concentration of drug within each vessel is given by $\theta_{\mathrm{adm}}(1-H)$ where $\theta_{\mathrm{adm}}$ is the administered dose. As with the oxygen, we view the vessels as distributed sources of drug and assume that, once the drug leaves the vessels, it diffuses through the tissue and is taken up by the normal and healthy cells.

Guided by equation (A1) and denoting by $\theta$ the drug concentration in the tissue, at each time step we solve the following diffusion equation, with no-flux boundary conditions, to determine $\theta$ :

$$
0=D_{\theta} \nabla^{2} \theta+h_{\theta}\left(\theta_{\text {vess }}-\theta\right)-\lambda_{\text {drug }} \theta
$$

In equation (A13), $D_{\theta}$ denotes the assumed constant diffusion coefficient of the drug, $h_{\theta}$ the rate at which it is transported across the vessel wall and $\lambda_{\text {drug }}$ the assumed constant rate at which cells absorb the drug (we assume that $\lambda_{\text {drug }}$ is the same for normal and cancerous cells).

Having determined $\theta_{\text {vess }}$ and $\theta$, it remains to specify the drug's mode of action. Ultimately, we hope to tailor our model to specific drugs according to the stage(s) of the cell cycle at which they act. However, for simplicity, here we assume that the drug works in the following manner. If the local drug concentration exceeds a threshold value then the cell is killed.

\section{A.5 Numerical algorithm}

When performing simulations the following algorithm is used at each time step:

i) update the vascular network using the structural adaptation rules;

ii) calculate the blood flow and haematocrit at all points within the vascular network;

iii) update the oxygen, VEGF and drug profiles by solving the relevant boundary value problems; and

iv) update the elements of the cellular automaton, and the intracellular variables associated with each non-empty element according to whether it is occupied by a normal or cancerous cell.

\section{B Parameter values}

In this Appendix, we provide the values of the parameters needed to solve the PDEs for the drugs (cytotoxic and anti-VEGFR drugs) as well as the values of the parameters corresponding to the up- and down-stream stimuli of the vascular adaptation (table A1). Other parameter values can be found in Ref. [3] and are reproduced here for completeness (tables A2 and A3). 


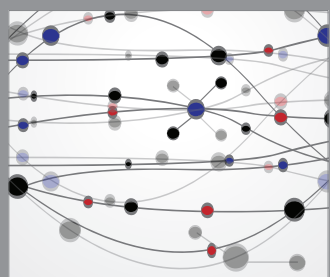

The Scientific World Journal
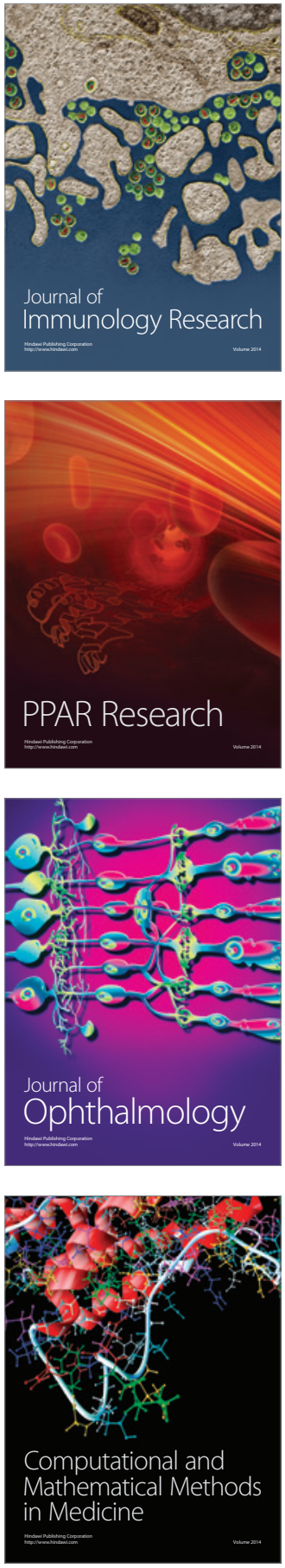

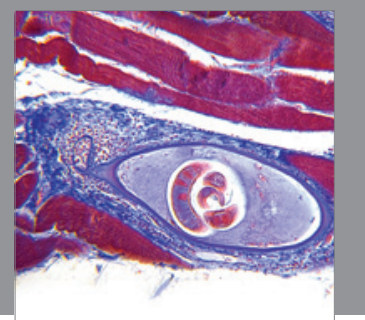

Gastroenterology

Research and Practice
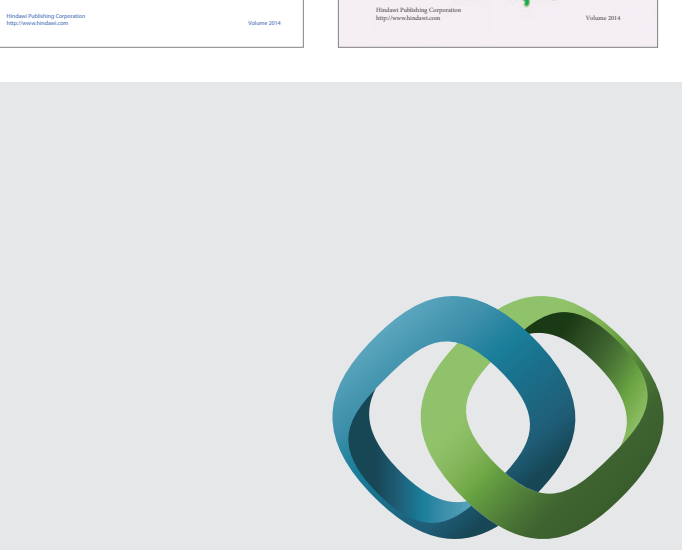

\section{Hindawi}

Submit your manuscripts at

http://www.hindawi.com
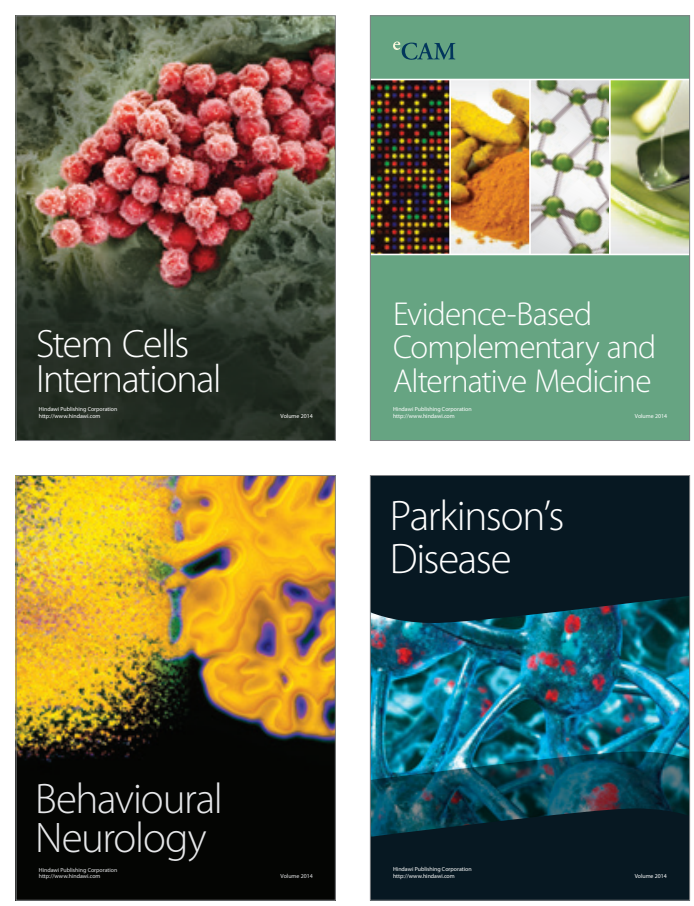

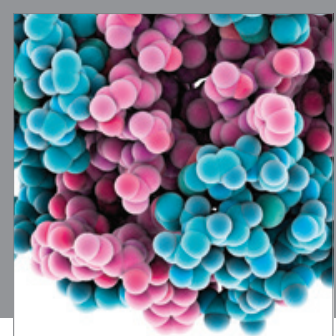

Journal of
Diabetes Research

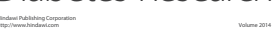

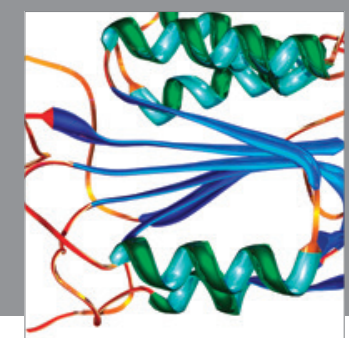

Disease Markers
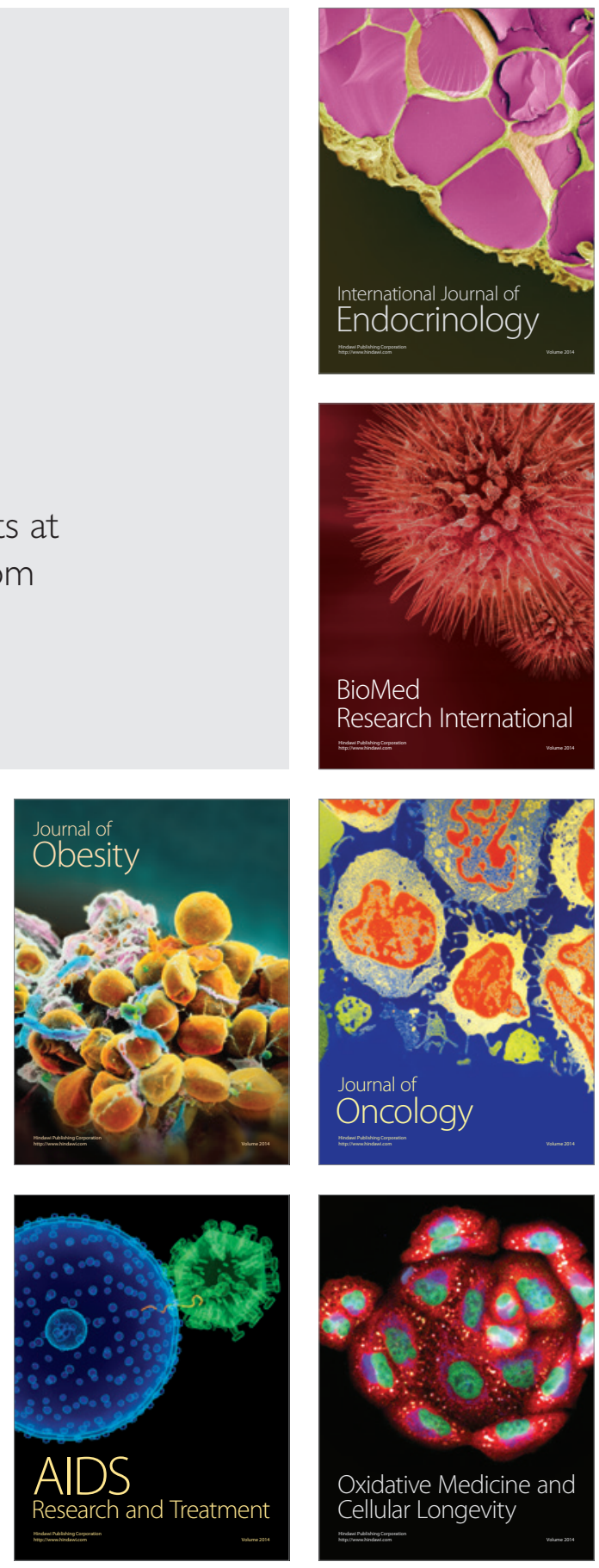